\title{
Insights on the crustal evolution of the West African Craton from Hf isotopes in detrital zircons from the Anti-Atlas belt
}

\author{
Jacobo Abati ${ }^{a, *}$, Abdel Mohsine Aghzer ${ }^{b, 1}{ }^{\text {, Axel Gerdes }}{ }^{c, d, 2}$, Nasser Ennih ${ }^{b}$

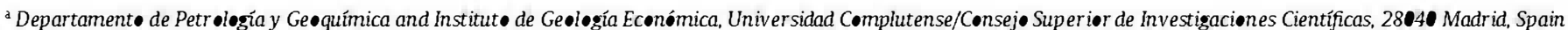

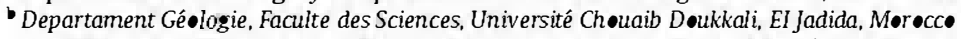

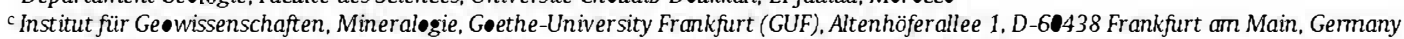 \\ d Department of Earth Sciences, Stellenb•sch University, Private Bag X1. Matieland 7602. Søuth Africa
}

Keywords:

Anti-Atlas belt

Morocco

Hfisotopes

Detrital zircon

Crustal evolution

\begin{abstract}
A B S T R A C T

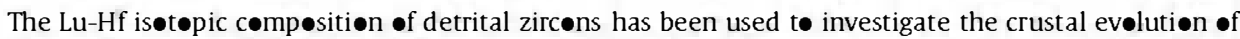

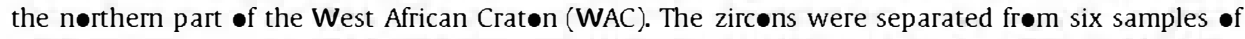

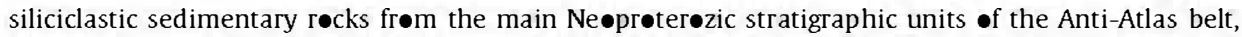
frøm the Sirwa and Zenaga inliers. The data suggest that the nørth part $\bullet$ the WAC førmed during three

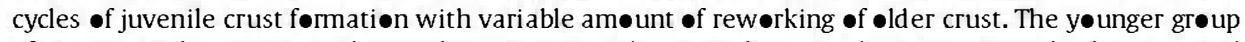

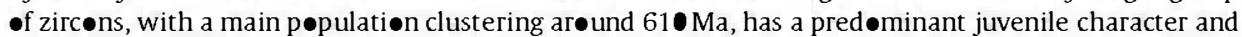

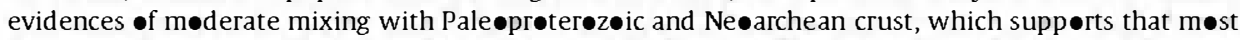

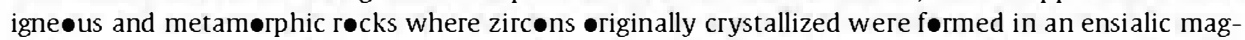

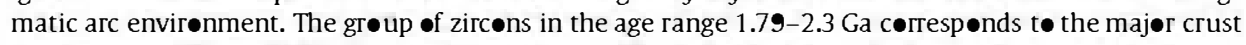

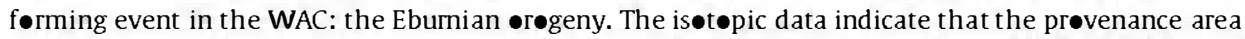
shøuld represent a crustal dømain that was separated frøm a mantle reserv ir at $\sim 205 \bullet-2300 \mathrm{Ma}$, and

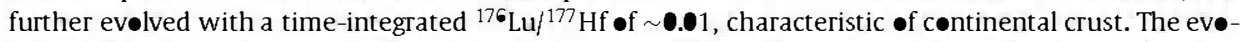

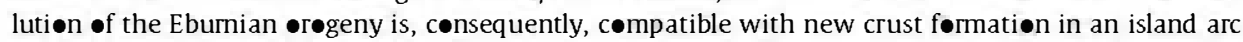

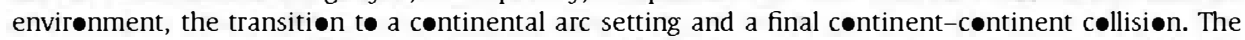

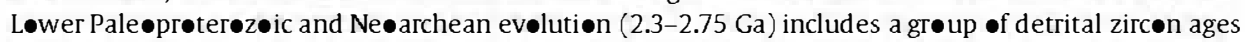

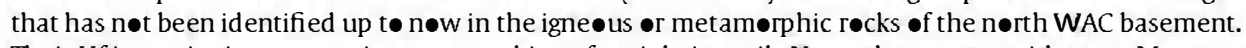

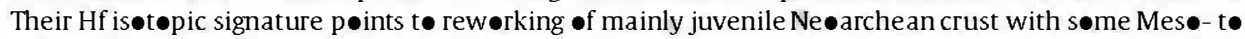
Palaearchean cøntributi $\bullet$ s. The significance of these ages is uncertain: they can represent a tectøn-ther-

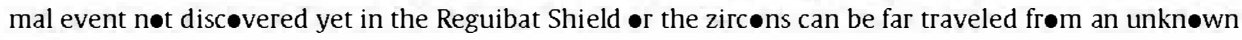
søurce.
\end{abstract}

\section{Introduction}

Lu-Hf isotopic investigations in single zircon grains of known age provides valuable information about the formation and evolution of the continental crust. Deviation of the $\mathrm{Hf}$ isotopic composition of one sample from the chondritic uniform reservoir (CHUR), usually reported as epsilon units, is a sensitive indicator of the source of the magmas where zircons formed, providing an estimation of the relative influence of the different components involved in the melting. Essentially, these components are juvenile depleted mantle, reworked old crust or a mixing of both. With the increasing availability and improved precision of analytical techniques, especially in situ zircon analyses, the Hf isotopic signature combined with the U-Pb age obtained in detrital or magmatic zircon grains has been widely used to constrain the mantle differentiation, crustal growth events and the nature of crustal reworking in many cratonic domains (Patchett et al., 1982; Stevenson and Patchett, 1990; Amelin et aI., 2000; Andersen et aI., 2002; Griffin et aI., 2004; Zhang et al., 2006; Gerdes and Zeh, 2006; Nebel-Jacobsen et al., 2010; Morag et aI., 2011). In this study we present LA-ICP-MS Lu$\mathrm{Hf}$ analyses of detrital zircons from six samples of Neoproterozoic sedimentary rocks from the Pan-African Anti-Atlas belt of southern Morocco, to investigate the crustal evolution of their proposed source area, in the northern part of the West African Craton (WAC). The old cratonic nuclei of the north part of the WAC is the Reguibat shield, formed by Paleoproterozoic and Archean components (Key et al., 2008), which is separated from the Anti-Atlas belt, more to the 


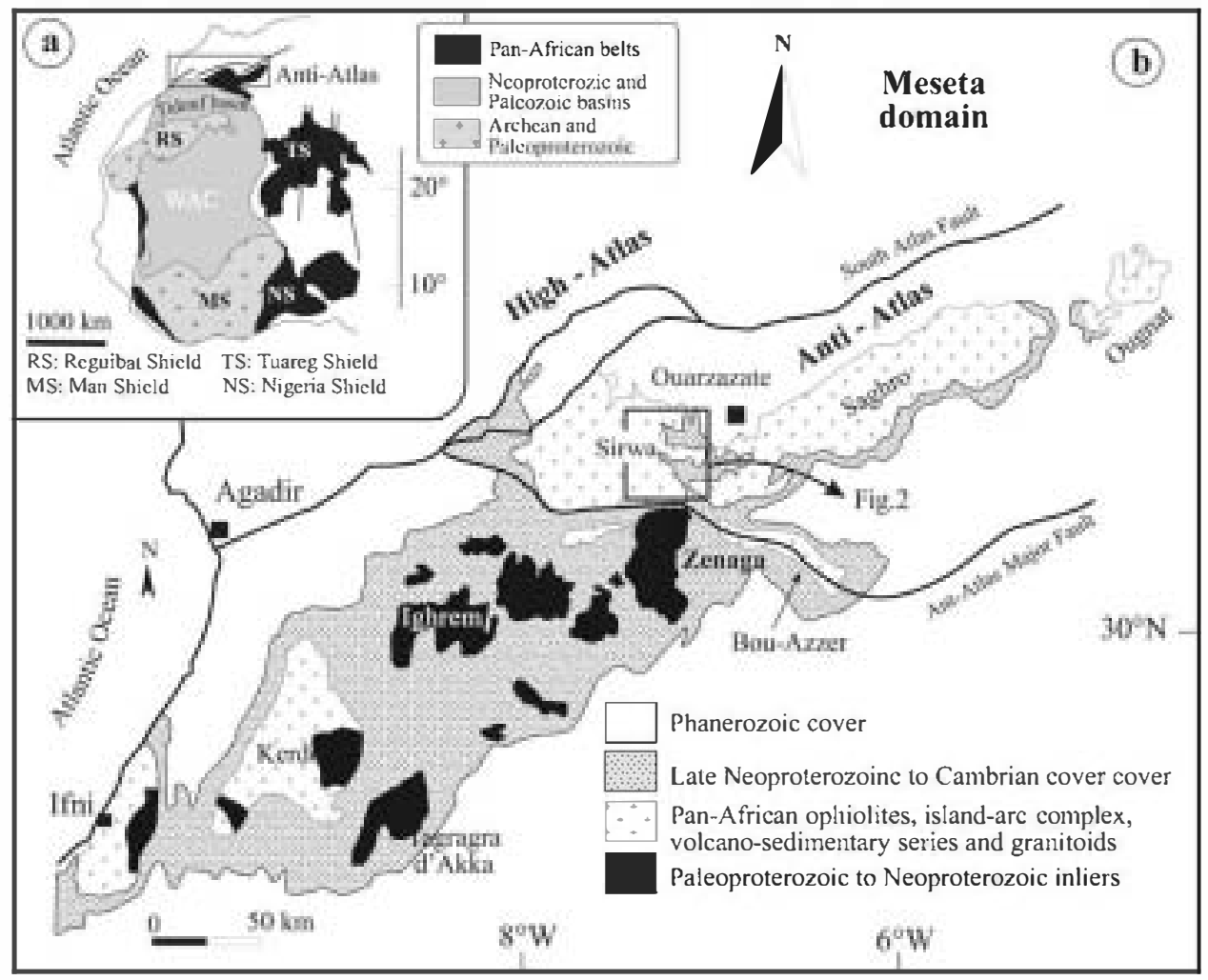

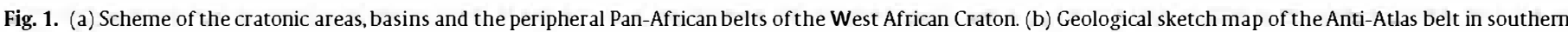
Morocco.

Based on Abati et al. (2010).

north, by the Tindouf basin (Fig. 1). The Anti-Atlas represents the remobilization of the northern cratonic boundary during the PanAfrican orogeny (Ennih and Liegeois, 2008), including arc accretion and ophiolite obduction to the margin. The older basement of the Anti-Atlas is formed by Paleoproterozoic Eburnian (2.0-2.2 Ga) magmatic and metamorphic rocks, covered by Neoproterozoic to Paleozoic sedimentary sequences. According to Abati et al. (2010), the major source of Neoproterozoic sediments (pre to post PanAfrican) was the Reguibat shield. For that reason, the detrital zircon populations from these sedimentary rocks are probably a representative sample of the main magmatic and metamorphic rocks from the north part of the WAC, and their isotopic composition can provide information about the main crustal components involved and their evolution.

\section{Geological setting}

\subsection{West African Craton}

The available age and isotopic data indicate that the building and amalgamation of the WAC basement took place through four major orogenic cycles: (1) Leonian cycle $(3.5-3.0 \mathrm{Ga}$; Rocci et al., 1991; Kröner et al., 2001; Potrel et al., 1996; Thiéblemont et al., 2004), (2) the Liberian cycle (2.95-2.75 Ga; Hurley et al., 1971; Auvray et al., 1992; Potrel et al., 1998; Key et al., 2008), (3) the Eburnian-Birimian cycle $(2.2-1.75 \mathrm{Ga}$; Abouchami et al., 1990; Liégeois et al., 1991; Boher et al., 1992; Hirdes et al., 1992; Ait Malek et al., 1998; Schofield et al., 2006) and (4) the PanAfrican remobilization (760-660 Ma; Leblanc and Lancelot, 1980; Saquaque et al., 1989; Hefferan et al., 2000; Thomas et al., 2002; Samson et al., 2004). One of the main characteristics of the WAC is that no Mesoproterozoic events or rocks are known, suggesting a quiescent period between 1.7 and $1.0 \mathrm{Ga}$ (e.g. Ennih and Liegeois, 2008). The exposed parts of the craton outcrop in two main uplifts: the Reguibat shield in the north (Mauritania, Morocco and Algeria) and the Leo Shield in the south (Fig. 1).

\subsection{Anti-Atlas belt}

The Anti-Atlas belt of southern Morocco is located on the northern edge of the West African Craton (WAC) (Fig. 1a). It is separated in the north from the High-Atlas and the Meseta domain by the South Atlas Fault (Fig. 1b). This belt is composed essentially by: (1) Basement rocks of Paleoproterozoic age (ca. $2 \mathrm{Ga}$ ); (2) Neoproterozoic sequences with lowermost units involved in the Pan African orogeny and (3) unconformably overlying Paleozoic rocks.

The Paleoproterozoic basement and overlying Pan-African Neoproterozoic sequences outcrops in several inliers (Ifni, Tagrara d’Akka, Kerdous, Sirwa, Ighrem, Zenaga, Iguerda, Bou Azzer, etc.) beneath post-Pan-African collisional cover consisting of late Neoproterozoic to Cambrian rocks (Fig. 1 b).

The Paleoproterozoic rocks (2030-2200 Ma) (Ait Malek et al., 1998; Thomas et al., 2002; Walsh et al., 2002) correspond to the northward margin of the WAC and consists essentially of low to high-grade metasediments intruded by calc-alkaline plutonic rocks. They have age-similarities with the West African Birimian crust.

The Neoproterozoic sequences involved in Pan-African orogeny are associated with a series of geodynamic process that summarized as follows:

1) The break-up and rifting of the northern edge of the WAC, leading to the formation of a passive margin sequence (The Taghdout Group) deposited on the Paleoproterozoic basement. 
2) The creation of an oceanic basin (760-697 Ma) (Samson et al., 2004; El Hadi et al., 2010) between the northern edge of the WAC and an unknown northern terrane. The preserved relic of this oceanic crust corresponds to highly sheared allochtonous ophiolite complexes (The Bou Azzer Group).

3) A subduction phase and island-arc formation (The Iriri Group) (743 Ma) (Thomas et al., 2002). A magmatic eventat 750-700 Ma (D'Lemos et al., 2006) recorded in the continental margin tectonically underlying the Taghdout Group in Bou Azzer inlier is interpreted as the result of pre-Pan-African rifting evolution (El Hadi et al., 2010) or as a continuous arc building event by Gasquet et al. (2008).

4) The ophiolite obduction, arc accretion onto the northern edge of the WAC and syn-tectonic calc-alkaline intrusions (660-635 Ma) (Thomas et al., 2002; Inglis et al., 2004; El Hadi et al., 2010).

5) A late to post-collisional Neoproterozoic evolution marked by the deposition of the Saghro Group, the Bou Salda Group and the emplacement of high-K-calc-alkaline granitoids of the Assarag suite. The Saghro Group consists of a volcanoclastic rocks metamorphosed at greenschist-facies conditions. The Bou Salda Group is a volcanosedimentary sequence that is preserved from the Pan-African metamorphism. The age of this group (620-610 Ma; Abati et al., 2010) is the same as the Saghro Group (630-610Ma) (Abati et al., 2010) and is indistinguishable from the age of 615-579 Ma (Aït Malek et al., 1998; De Wall et al., 2001; Levresse et al., 2001; Thomas et al., 2002; Inglis et al., 2004) of some granitoid intrusions of the Assarag suite (Abati et al., 2010). This synchronicity of the sedimentation and subsequent metamorphism, deformation and intrusion by plutonic bodies is a typical feature of magmatic arc settings (Abati et al., 2003, 2010). It suggests that the Saghro and Bou Salda Groups come from the erosion dismantling of a magmatic arc located to the north of the Pan-African suture and built upon north WAC basement (Abati et al., 2010). This arc-setting model can be correlated with the late Neoproterozoic (ca. 640-570 Ma) longlived arc-system characteristic of the north Gondwana margin and peri-Gondwanan terranes. The post-630-610 Ma deformation (and ante-580Ma: age of intrusive rocks of the overlying discordant Ouarzazate Group) affecting essentially the Saghro Group corresponds to the late phase of Pan-African collision (Abati et al., 2010).

The late Neoproterozoic evolution of the Anti-Atlas belt is marked by the deposition of the volcano-sedimentary rocks of the Ouarzazate Group (577-552 Ma) (Thomas et al., 2002; Walsh et al., 2002; Gasquet et al., 2005; Abati et al., 2010) that rest unconformably on the Paleoproterozoic basement and the Neoproterozoic units involved in the Pan-African orogeny.

\section{Sample description}

Six sedimentary rocks were sampled from the Taghdout, Saghro, Bou Salda (Sirwa inlier) and Ouarzazate groups (Zenaga inlier), encompassing the major units of the Neoproterozoic sequences of the Anti-Atlas (Fig. 2). Following a bottom to top order, sample AA1 is an undeformed quartzite of the Mimount Formation, collected from the type locality of Taghdout village, belonging to the Taghdout Group, interpreted as the oldest rocks overlying the WAC (Thomas et al., 2002; 740-800 Ma). Three samples are from Saghro Group: AA5 is a arkose and AA3 is a conglomerate (diamictite) from the Imghi Formation, whereas AA6 is an arkosic sandstone from the Azarwas Formation. Sample AA4 is a quartzic cobble of a conglomerate from the Lmakhzane Member of the Bou Salda Group. Finally, sample AA7 is an epiclastic conglomerate of the Ouarzazate Group. The location of the samples is showed in the map and in the schematic stratigraphic column of Fig. 2. They are the same samples of the U-Pb study of Abati et al., 2010, and more detailed descriptions, maps and stratigraphic features can be found there (Table 1).

\subsection{Analytical techniques}

Hafnium isotope measurements were performed with ThermoScientific Neptune multi-collector ICP-MS coupled to a New Wave Research UP213 ultraviolet laser system at Goethe-University Frankfurt (GUF) following the method described by Gerdes and Zeh (2006, 2009). A teardrop-shaped, low volume $\left(<2.5 \mathrm{~cm}^{3}\right)$ laser cell with fast response ( $<1 \mathrm{~s}$ ) and low wash-out time was used (Janousek et al., 2006; Frei and Gerdes, 2009). The Lu-Hf laser spots ( $40 \mu \mathrm{m}$ in diameter) were drilled on top of the $30 \mu \mathrm{m}$ spots used for U-Pb analyses.

Data were collected in static mode during $58 \mathrm{~s}$ of laser ablation. Nitrogen $(\sim 0.005 \mathrm{I} / \mathrm{min})$ was introduced via a Cetac Aridus into the Ar sample carrier gas to enhance sensitivity ( 30\%) and to reduce oxide formation. The use of the Aridus system allowed bracketing of laser ablation analyses with solution mode analyses. Analytical protocols were the same for laser ablation and solution mode analyses. The isotopes ${ }^{172} \mathrm{Yb},{ }^{173} \mathrm{Yb}$ and ${ }^{175} \mathrm{Lu}$ were simultaneously monitored during each analysis step to allow for correction of isobaric interferences of $\mathrm{Lu}$ and $\mathrm{Yb}$ isotopes on mass 176. The ${ }^{176} \mathrm{Lu}$ and ${ }^{176} \mathrm{Yb}$ were calculated using ${ }^{176} \mathrm{Lu} /{ }^{175} \mathrm{Lu}$ of 0.02658 and ${ }^{176} \mathrm{Yb} /{ }^{173} \mathrm{Yb}$ of 0.795015 (both GUF in-house values). The accuracy of the correction was tested with a 40 ppb JMC475 solution doped with natural Yb and Lu. Even at about $100 \%$ interference the corrected yielded a ${ }^{176} \mathrm{Hf} /{ }^{177} \mathrm{Hf}$ of $0.282157 \pm 40$ (2SD, Table S1). For a more detailed discussion see Gerdes and Zeh (2009). All data were adjusted relative to the JMC475 of ${ }^{176} \mathrm{Hf} /{ }^{177} \mathrm{Hf}$ ratio $=0.282160$ and quoted uncertainties are quadratic additions of the within run precision of each analysis and the reproducibility of the JMC $475(2 \mathrm{SD}=0.0033 \%, n=8$; Table S1). Accuracy and external reproducibility of the method was verified by repeated analyses of reference zircon GJ-1 and Plešovice, which yielded a ${ }^{176} \mathrm{Hf} /{ }^{177} \mathrm{Hf}$ of $0.282003 \pm 0.000023$ (2SD, $n=23$ ) and of $0.282469 \pm 0.000023$ $(n=15)$, respectively (Table S1).

\section{Hf isotopes and WAC crustal evolution}

The detrital zircons of this study can be grouped in two major age populations (Fig. 3a), pointing to the existence of two periods of maximum granitoid production: a younger Neoproterozoic group ranging from 540 to $750 \mathrm{Ma}$ (syn to post Pan-African collision) and an older Paleoproterozoic to Archean group ranging from 1790 to $2750 \mathrm{Ma}$. The two groups are separated by an age gap between 1.7 and $0.8 \mathrm{Ma}$, characteristic of the WAC (Ennih and Liegeois, 2008). The older group can in turn be separated in two different groups, according to their isotopic features (1790-2300 and 2300-2750 Ma).

\subsection{Neoproterozoic zircons: a pan-african arc in the periphery of gondwana?}

The zircons show a wide variability of the initial epsilon Hf $\left(\varepsilon \mathrm{Hf}_{\mathrm{i}}\right.$ ) values, ranging between -14 and 14 (Fig. 3a and b). From the 111 zircons analyzed of this age range, about $70 \%$ have positive $\varepsilon \mathrm{Hf}_{\mathrm{i}}$, indicating that they were crystallized either from magmas directly derived from a depleted mantle source (=juvenile magmas) contaminated with different amounts of older crust or by partial melting of a relatively juvenile crust, e.g. a recently formed island arc. In any case, the data suggest that significant amounts of juvenile crust were formed during late Neoproterozoic time. The 
Table 1

Lu-Hf isotopic data.

\begin{tabular}{|c|c|c|c|c|c|c|c|c|c|c|c|c|c|c|c|}
\hline & ${ }^{17} \cdot \mathrm{Yb} / /^{177} \mathrm{Hf}^{\mathrm{a}}$ & $\pm 2 \sigma$ & ${ }^{17} \cdot \mathrm{Lu} / /^{177} \mathrm{Hf}^{a}$ & $\pm 2 \sigma$ & ${ }^{178} \mathrm{Hf} /{ }^{177} \mathrm{Hf}$ & ${ }^{180} \mathrm{Hf} /{ }^{177} \mathrm{Hf}$ & $\operatorname{Sig}_{\mathrm{Hf}}(\mathrm{V})$ & ${ }^{17} \mathrm{Hf}^{1777} \mathrm{Hf}^{\mathrm{d}}$ & $\pm 2 \sigma^{c}$ & ${ }^{17} \cdot \mathrm{Hf} /{ }^{177} \mathrm{Hf}_{(t)}$ & $\varepsilon H f(t)^{d}$ & $\pm 2 \sigma^{c}$ & $\mathrm{~T}_{\mathrm{M} 2} e^{e}(\mathrm{Ga})$ & $\operatorname{Age}^{\mathrm{f}}(\mathrm{Ma})$ & $\pm 2 \sigma$ \\
\hline AA1_1 & 0.0296 & 48 & 0.00112 & 14 & 1.46730 & 1.88686 & 14 & 0.281581 & 28 & 0.281534 & 5.7 & 1.0 & 2.19 & 2202 & 17 \\
\hline AA 1.2 & 0.0312 & 35 & 0.00088 & 9 & 1.46734 & 1.88680 & 13 & 0.281626 & 25 & 0.281590 & 6.4 & 0.9 & 2.11 & 2149 & 12 \\
\hline AA 1 _3 & 0.0427 & 47 & 0.00112 & 11 & 1.46733 & 1.88687 & 12 & 0.281628 & 27 & 0.281583 & 4.8 & 1.0 & 2.15 & 2091 & 21 \\
\hline AA1_5 & 0.0323 & 31 & 0.00087 & 7 & 1.46711 & 1.88656 & 16 & 0.281670 & 33 & 0.281636 & 6.2 & 1.2 & 2.05 & 2069 & 34 \\
\hline AA1_6 & 0.0223 & 22 & 0.00063 & 5 & 1.46733 & 1.88671 & 16 & 0.281551 & 22 & 0.281528 & -0.9 & 0.8 & 2.33 & 1927 & 27 \\
\hline AA 1.7 & 0.0164 & 39 & 0.00047 & 10 & 1.46739 & 1.88670 & 19 & 0.281621 & 21 & 0.281601 & 8.0 & 0.8 & 2.06 & 2200 & 29 \\
\hline AA1_9 & 0.0212 & 18 & 0.00058 & 4 & 1.46717 & 1.88651 & 13 & 0.281431 & 24 & 0.281407 & 1.2 & 0.8 & 2.44 & 2206 & 16 \\
\hline AA 1.11 & 0.0772 & 143 & 0.00205 & 39 & 1.46736 & 1.88685 & 21 & 0.281668 & 41 & 0.281587 & 4.7 & 1.5 & 2.14 & 2079 & 12 \\
\hline AA1_12 & 0.0632 & 120 & 0.00185 & 33 & 1.46729 & 1.88682 & 18 & 0.281681 & 50 & 0.281604 & 7.6 & 1.8 & 2.06 & 2178 & 23 \\
\hline AA $1 \_16$ & 0.0107 & 13 & 0.00035 & 3 & 1.46721 & 1.88665 & 20 & 0.281329 & 16 & 0.281313 & 2.9 & 0.6 & 2.52 & 2420 & 30 \\
\hline AA1_17 & 0.0485 & 77 & 0.00145 & 21 & 1.46733 & 1.88677 & 13 & 0.281392 & 33 & 0.281331 & -1.6 & 1.2 & 2.59 & 2199 & 24 \\
\hline AA1_18 & 0.0385 & 43 & 0.00109 & 12 & 1.46734 & 1.88672 & 14 & 0.280991 & 27 & 0.280933 & -3.0 & 1.0 & 3.11 & 2746 & 30 \\
\hline AA $1 \_20$ & 0.0294 & 25 & 0.00090 & 6 & 1.46733 & 1.88687 & 14 & 0.281514 & 30 & 0.281479 & 0.7 & 1.1 & 2.36 & 2075 & 26 \\
\hline AA127 & 0.0124 & 10 & 0.00036 & 2 & 1.46718 & 1.88658 & 13 & 0.281648 & 21 & 0.281634 & 6.3 & 0.8 & 2.05 & 2077 & 24 \\
\hline AA1.28 & 0.0200 & 19 & 0.00054 & 4 & 1.46721 & 1.88671 & 13 & 0.281354 & 28 & 0.281328 & 4.4 & 1.0 & 2.47 & 2462 & 19 \\
\hline AA1_30 & 0.0205 & 20 & 0.00051 & 4 & 1.46731 & 1.88677 & 13 & 0.281580 & 19 & 0.281557 & 8.4 & 0.7 & 2.11 & 2283 & 27 \\
\hline AA1_32 & 0.0271 & 37 & 0.00074 & 8 & 1.46724 & 1.88679 & 14 & 0.281288 & 25 & 0.281253 & 2.9 & 0.9 & 2.60 & 2514 & 16 \\
\hline AA1_33 & 0.0635 & 117 & 0.00147 & 23 & 1.46722 & 1.88653 & 15 & 0.281652 & 39 & 0.281593 & 5.1 & 1.4 & 2.13 & 2087 & 13 \\
\hline AA1_37 & 0.0201 & 29 & 0.00048 & 7 & 1.46730 & 1.88689 & 17 & 0.281600 & 18 & 0.281583 & 1.5 & 0.6 & 2.21 & 1948 & 11 \\
\hline AA1_41 & 0.0238 & 25 & 0.00075 & 6 & 1.46714 & 1.88650 & 16 & 0.281537 & 21 & 0.281507 & 2.3 & 0.8 & 2.29 & 2100 & 16 \\
\hline AA1_42 & 0.0159 & 13 & 0.00043 & 3 & 1.46724 & 1.88672 & 13 & 0.281204 & 20 & 0.281186 & -6.6 & 0.7 & 2.87 & 2205 & 26 \\
\hline AA1_43 & 0.0262 & 21 & 0.00074 & 5 & 1.46724 & 1.88681 & 14 & 0.281404 & 21 & 0.281371 & 3.2 & 0.8 & 2.44 & 2343 & 14 \\
\hline AA1_45 & 0.0496 & 50 & 0.00156 & 17 & 1.46733 & 1.88682 & 19 & 0.281480 & 27 & 0.281411 & 4.0 & 1.0 & 2.38 & 2319 & 22 \\
\hline AA1_46 & 0.0410 & 34 & 0.00113 & 8 & 1.46731 & 1.88676 & 17 & 0.281647 & 26 & 0.281603 & 4.7 & 0.9 & 2.12 & 2055 & 18 \\
\hline AA1_47 & 0.0081 & 11 & 0.00020 & 3 & 1.46731 & 1.88689 & 19 & 0.281486 & 20 & 0.281478 & 0.8 & 0.7 & 2.36 & 2080 & 17 \\
\hline AA1_52 & 0.0102 & 10 & 0.00030 & 2 & 1.46717 & 1.88648 & 15 & 0.281287 & 18 & 0.281273 & 1.7 & 0.7 & 2.60 & 2432 & 33 \\
\hline AA1_.55 & 0.0230 & 20 & 0.00081 & 6 & 1.46715 & 1.88663 & 13 & 0.281591 & 20 & 0.281562 & -0.2 & 0.7 & 2.27 & 1905 & 29 \\
\hline AA1.57 & 0.0222 & 20 & 0.00060 & 4 & 1.46731 & 1.88687 & 14 & 0.281599 & 20 & 0.281576 & 2.6 & 0.7 & 2.20 & 2006 & 27 \\
\hline AA1_58 & 0.0334 & 28 & 0.00084 & 5 & 1.46722 & 1.88654 & 13 & 0.281386 & 24 & 0.281346 & 4.6 & 0.8 & 2.45 & 2443 & 20 \\
\hline AA1_60 & 0.0168 & 14 & 0.00045 & 3 & 1.46733 & 1.88687 & 14 & 0.281527 & 18 & 0.281511 & -3.1 & 0.6 & 2.40 & 1857 & 24 \\
\hline AA1_61 & 0.0688 & 66 & 0.00166 & 14 & 1.46718 & 1.88656 & 17 & 0.281484 & 37 & 0.281418 & -0.7 & 1.3 & 2.47 & 2105 & 17 \\
\hline AA 1_64 & 0.0697 & 60 & 0.00162 & 10 & 1.46719 & 1.88652 & 19 & 0.281092 & 38 & 0.281030 & -16.3 & 1.3 & 3.26 & 2028 & 14 \\
\hline AA1_65 & 0.0205 & 18 & 0.00051 & 3 & 1.46730 & 1.88668 & 13 & 0.281651 & 18 & 0.281623 & 26.0 & 0.7 & 1.64 & 2936 & 15 \\
\hline AA1_66 & 0.0389 & 73 & 0.00105 & 23 & 1.46733 & 1.88677 & 14 & 0.281096 & 27 & 0.281055 & -13.8 & 1.0 & 3.18 & 2096 & 16 \\
\hline AA1.70 & 0.0346 & 35 & 0.00083 & 6 & 1.46715 & 1.88649 & 13 & 0.281666 & 23 & 0.281634 & 4.8 & 0.8 & 2.08 & 2013 & 19 \\
\hline AA1_72 & 0.0670 & 66 & 0.00169 & 15 & 1.46732 & 1.88679 & 17 & 0.281685 & 33 & 0.281618 & 6.0 & 1.2 & 2.08 & 2090 & 19 \\
\hline AA1_.73 & 0.0668 & 71 & 0.00180 & 19 & 1.46717 & 1.88647 & 15 & 0.281468 & 26 & 0.281392 & 1.0 & 0.9 & 2.46 & 2220 & 23 \\
\hline AA1_74 & 0.0471 & 46 & 0.00119 & 8 & 1.46716 & 1.88658 & 12 & 0.281604 & 26 & 0.281557 & 3.6 & 0.9 & 2.21 & 2077 & 23 \\
\hline AA1_80 & 0.0331 & 28 & 0.00082 & 6 & 1.46725 & 1.88683 & 16 & 0.281229 & 21 & 0.281196 & -8.5 & 0.8 & 2.90 & 2110 & 19 \\
\hline AA1_.81 & 0.0755 & 67 & 0.00187 & 15 & 1.46725 & 1.88662 & 19 & 0.281700 & 36 & 0.281628 & 4.8 & 1.3 & 2.09 & 2021 & 10 \\
\hline AA1_84 & 0.0245 & 22 & 0.00061 & 4 & 1.46713 & 1.88656 & 17 & 0.281612 & 24 & 0.281589 & 3.4 & 0.8 & 2.17 & 2020 & 15 \\
\hline AA1_.87 & 0.0428 & 58 & 0.00095 & 15 & 1.46712 & 1.88658 & 15 & 0.281114 & 34 & 0.281076 & -12.8 & 1.2 & 3.13 & 2108 & 20 \\
\hline AA1_89 & 0.0476 & 52 & 0.00130 & 10 & 1.46735 & 1.88676 & 12 & 0.281200 & 29 & 0.281137 & -0.6 & 1.0 & 2.81 & 2538 & 13 \\
\hline AA1_90 & 0.0739 & 72 & 0.00186 & 13 & 1.46719 & 1.88656 & 19 & 0.281397 & 38 & 0.281324 & -5.0 & 1.4 & 2.67 & 2064 & 11 \\
\hline AA1_93 & 0.0255 & 21 & 0.00067 & 5 & 1.46719 & 1.88657 & 15 & 0.281566 & 20 & 0.281539 & 3.6 & 0.7 & 2.23 & 2105 & 19 \\
\hline AA1_97 & 0.0232 & 27 & 0.00062 & 5 & 1.46718 & 1.88657 & 17 & 0.281267 & 20 & 0.281238 & 2.7 & 0.7 & 2.62 & 2527 & 15 \\
\hline AA 1.104 & 0.0031 & 13 & 0.00007 & 3 & 1.46723 & 1.88682 & 15 & 0.281361 & 15 & 0.281358 & -3.3 & 0.5 & 2.59 & 2087 & 13 \\
\hline AA 1_106 & 0.0031 & 13 & 0.00007 & 3 & 1.46723 & 1.88682 & 15 & 0.281361 & 15 & 0.281358 & -2.3 & 0.5 & 2.57 & 2129 & 11 \\
\hline AA 1_109 & 0.0493 & 42 & 0.00120 & 8 & 1.46726 & 1.88667 & 12 & 0.281580 & 30 & 0.281539 & -3.3 & 1.1 & 2.36 & 1809 & 15 \\
\hline AA 1.110 & 0.0203 & 22 & 0.00059 & 6 & 1.46734 & 1.88684 & 16 & 0.281041 & 20 & 0.281011 & -4.6 & 0.7 & 3.05 & 2561 & 10 \\
\hline AA1_111 & 0.0153 & 17 & 0.00051 & 4 & 1.46731 & 1.88663 & 14 & 0.281380 & 23 & 0.281358 & 2.2 & 0.8 & 2.48 & 2322 & 12 \\
\hline AA 1.116 & 0.0372 & 33 & 0.00106 & 7 & 1.46724 & 1.88673 & 16 & 0.281364 & 27 & 0.281315 & 2.3 & 1.0 & 2.53 & 2392 & 9 \\
\hline AA 1.120 & 0.0204 & 17 & 0.00062 & 5 & 1.46729 & 1.88686 & 14 & 0.281304 & 22 & 0.281274 & 3.7 & 0.8 & 2.55 & 2513 & 10 \\
\hline AA 1.125 & 0.0247 & 20 & 0.00069 & 4 & 1.46727 & 1.88677 & 11 & 0.281529 & 26 & 0.281504 & -0.8 & 0.9 & 2.36 & 1969 & 30 \\
\hline AA 1.127 & 0.0436 & 40 & 0.00105 & 9 & 1.46723 & 1.88667 & 9 & 0.281502 & 31 & 0.281465 & -5.5 & 1.1 & 2.50 & 1825 & 15 \\
\hline
\end{tabular}




\begin{tabular}{|c|c|c|c|c|c|c|c|c|c|c|c|c|c|c|c|}
\hline & ${ }^{17} \mathrm{Yb}^{17{ }^{177}} \mathrm{Hf}^{\mathrm{a}}$ & $\pm 2 \sigma$ & ${ }^{17} \mathrm{Lu}^{1777} \mathrm{Hf}^{\mathrm{d}}$ & $\pm 2 \sigma$ & ${ }^{178} \mathrm{Hf} /{ }^{177} \mathrm{Hf}$ & ${ }^{180} \mathrm{Hf} /{ }^{177} \mathrm{Hf}$ & $\mathrm{Sig}_{\mathrm{Hf}}(\mathrm{V})$ & ${ }^{176} \mathrm{Hf}^{177} \mathrm{Hf}^{\mathrm{d}}$ & $\pm 2 \sigma^{c}$ & ${ }^{17} \mathbf{6}_{\mathrm{Hf}} /{ }^{177} \mathrm{Hf}_{\mathrm{ft}}$ & $\varepsilon \mathrm{Hf}(\mathrm{t})^{\mathrm{d}}$ & $\pm 2 \sigma^{c}$ & $\mathrm{~T}_{\mathrm{M} 2}{ }^{e}(\mathrm{Ga})$ & $\operatorname{Age}^{f}(\mathrm{Ma})$ & $\pm 2 \sigma$ \\
\hline AA3_1 & 0.0190 & 16 & 0.00053 & 4 & 1.46718 & 1.88648 & 12 & 0.282072 & 20 & 0.282066 & -11.9 & 0.7 & 1.85 & 608 & 9 \\
\hline AA3_3 & 0.0798 & 76 & 0.00194 & 14 & 1.46729 & 1.88676 & 14 & 0.282347 & 27 & 0.282326 & -3.3 & 1.0 & 1.36 & 579 & 9 \\
\hline AA3.5 & 0.0360 & 51 & 0.00094 & 12 & 1.46720 & 1.88683 & 15 & 0.282199 & 25 & 0.282189 & -7.8 & 0.9 & 1.62 & 598 & 9 \\
\hline AA3.7 & 0.0305 & 34 & 0.00085 & 9 & 1.46721 & 1.88652 & 12 & 0.281610 & 21 & 0.281579 & 0.4 & 0.7 & 2.24 & 1905 & 26 \\
\hline AA3_12 & 0.0245 & 20 & 0.00064 & 4 & 1.46715 & 1.88660 & 13 & 0.281665 & 20 & 0.281640 & 7.3 & 0.7 & 2.03 & 2111 & 17 \\
\hline AA 3-14 & 0.0504 & 41 & 0.00122 & 7 & 1.46726 & 1.88679 & 15 & 0.282307 & 28 & 0.282292 & -3.3 & 1.0 & 1.40 & 633 & 9 \\
\hline AA3.15 & 0.0447 & 37 & 0.00113 & 8 & 1.46720 & 1.88653 & 11 & 0.281180 & 27 & 0.281121 & 3.1 & 1.0 & 2.75 & 2722 & 14 \\
\hline AA3_16 & 0.0431 & 35 & 0.00109 & 7 & 1.46723 & 1.88673 & 15 & 0.282316 & 26 & 0.282303 & -3.4 & 0.9 & 1.39 & 612 & 10 \\
\hline AA3_17 & 0.0827 & 84 & 0.00187 & 13 & 1.46723 & 1.88666 & 15 & 0.282291 & 43 & 0.282270 & -4.8 & 1.5 & 1.46 & 603 & 11 \\
\hline AA3_18 & 0.0503 & 48 & 0.00130 & 10 & 1.46719 & 1.88657 & 11 & 0.281634 & 30 & 0.281582 & 5.1 & 1.1 & 2.14 & 2106 & 14 \\
\hline AA3_19 & 0.0335 & 28 & 0.00082 & 5 & 1.46720 & 1.88658 & 16 & 0.282070 & 21 & 0.282061 & -11.3 & 0.8 & 1.85 & 640 & 8 \\
\hline AA3_20 & 0.0700 & 88 & 0.00161 & 16 & 1.46724 & 1.88655 & 13 & 0.281714 & 38 & 0.281658 & 1.5 & 1.4 & 2.12 & 1830 & 34 \\
\hline AA3_21 & 0.0223 & 22 & 0.00066 & 6 & 1.46724 & 1.88661 & 15 & 0.281627 & 21 & 0.281600 & 7.3 & 0.8 & 2.07 & 2173 & 19 \\
\hline AA3_23 & 0.0265 & 23 & 0.00073 & 6 & 1.46729 & 1.88673 & 13 & 0.281616 & 21 & 0.281588 & 3.3 & 0.7 & 2.17 & 2016 & 15 \\
\hline AA3_24 & 0.0546 & 48 & 0.00140 & 10 & 1.46728 & 1.88672 & 15 & 0.281629 & 30 & 0.281573 & 4.2 & 1.1 & 2.17 & 2080 & 17 \\
\hline AA3_26 & 0.0517 & 52 & 0.00132 & 12 & 1.46719 & 1.88679 & 14 & 0.281637 & 31 & 0.281584 & 4.7 & 1.1 & 2.15 & 2085 & 15 \\
\hline AA3_27 & 0.1133 & 95 & 0.00281 & 18 & 1.46715 & 1.88654 & 11 & 0.282352 & 55 & 0.282317 & -2.2 & 2.0 & 1.35 & 644 & 11 \\
\hline AA3_28 & 0.0549 & 88 & 0.00135 & 19 & 1.46720 & 1.88663 & 13 & 0.281655 & 31 & 0.281603 & 4.7 & 1.1 & 2.13 & 2056 & 10 \\
\hline AA3_29 & 0.0256 & 22 & 0.00062 & 4 & 1.46713 & 1.88650 & 16 & 0.281588 & 22 & 0.281566 & -0.1 & 0.8 & 2.27 & 1907 & 13 \\
\hline AA3_31 & 0.0178 & 18 & 0.00054 & 4 & 1.46721 & 1.88662 & 15 & 0.281579 & 19 & 0.281558 & 4.8 & 0.7 & 2.18 & 2128 & 10 \\
\hline AA3_35 & 0.0166 & 13 & 0.00043 & 3 & 1.46718 & 1.88670 & 12 & 0.281125 & 20 & 0.281105 & -5.6 & 0.7 & 2.95 & 2374 & 21 \\
\hline AA3_36 & 0.0164 & 14 & 0.00049 & 3 & 1.46715 & 1.88650 & 14 & 0.282554 & 16 & 0.282548 & 6.4 & 0.6 & 0.89 & 662 & 12 \\
\hline AA3_37 & 0.0399 & 34 & 0.00105 & 6 & 1.46712 & 1.88652 & 12 & 0.282341 & 28 & 0.282329 & -2.9 & 1.0 & 1.35 & 593 & 10 \\
\hline AA3_38 & 0.0700 & 60 & 0.00161 & 10 & 1.46722 & 1.88663 & 12 & 0.282325 & 35 & 0.282306 & -3.0 & 1.2 & 1.38 & 624 & 12 \\
\hline AA3_39 & 0.0845 & 79 & 0.00196 & 14 & 1.46722 & 1.88659 & 13 & 0.282292 & 41 & 0.282270 & -4.6 & 1.5 & 1.45 & 612 & 12 \\
\hline AA3_40 & 0.0365 & 34 & 0.00090 & 7 & 1.46721 & 1.88657 & 11 & 0.282290 & 24 & 0.282280 & -4.3 & 0.9 & 1.43 & 611 & 11 \\
\hline AA3_41 & 0.0525 & 118 & 0.00126 & 27 & 1.46721 & 1.88662 & 13 & 0.282291 & 37 & 0.282276 & -3.8 & 1.3 & 1.43 & 637 & 11 \\
\hline AA3_43 & 0.0345 & 30 & 0.00104 & 7 & 1.46719 & 1.88651 & 13 & 0.282544 & 23 & 0.282532 & 4.8 & 0.8 & 0.94 & 613 & 10 \\
\hline AA3_44 & 0.0483 & 50 & 0.00132 & 11 & 1.46723 & 1.88667 & 11 & 0.281567 & 29 & 0.281512 & 4.7 & 1.0 & 2.24 & 2193 & 19 \\
\hline AA3_46 & 0.0797 & 68 & 0.00190 & 13 & 1.46728 & 1.88677 & 13 & 0.282309 & 37 & 0.282286 & -3.4 & 1.3 & 1.41 & 637 & 13 \\
\hline AA3_49 & 0.0537 & 62 & 0.00137 & 12 & 1.46720 & 1.88664 & 8 & 0.282312 & 30 & 0.282296 & -3.5 & 1.0 & 1.40 & 617 & 10 \\
\hline AA3_50 & 0.0747 & 66 & 0.00175 & 12 & 1.46720 & 1.88655 & 13 & 0.282323 & 39 & 0.282304 & -3.9 & 1.4 & 1.40 & 591 & 9 \\
\hline AA3_51 & 0.1180 & 107 & 0.00266 & 18 & 1.46714 & 1.88659 & 10 & 0.282503 & 53 & 0.282471 & 3.1 & 1.9 & 1.05 & 634 & 11 \\
\hline AA3_57 & 0.0714 & 59 & 0.00176 & 11 & 1.46731 & 1.88682 & 16 & 0.281604 & 33 & 0.281534 & 2.8 & 1.2 & 2.25 & 2078 & 12 \\
\hline AA3_58 & 0.0822 & 71 & 0.00214 & 15 & 1.46731 & 1.88684 & 13 & 0.282716 & 37 & 0.282688 & 12.5 & 1.3 & 0.59 & 714 & 12 \\
\hline AA3_62 & 0.0461 & 44 & 0.00115 & 8 & 1.46719 & 1.88655 & 14 & 0.282310 & 27 & 0.282297 & -4.1 & 0.9 & 1.41 & 591 & 12 \\
\hline AA3_66 & 0.0919 & 80 & 0.00202 & 13 & 1.46724 & 1.88681 & 12 & 0.282434 & 44 & 0.282411 & 0.3 & 1.6 & 1.18 & 606 & 11 \\
\hline AA3_67 & 0.0950 & 90 & 0.00224 & 17 & 1.46722 & 1.88679 & 12 & 0.282301 & 44 & 0.282275 & -4.3 & 1.6 & 1.44 & 618 & 11 \\
\hline AA3_69 & 0.0255 & 21 & 0.00073 & 5 & 1.46733 & 1.88665 & 13 & 0.282680 & 23 & 0.282672 & 9.8 & 0.8 & 0.66 & 616 & 12 \\
\hline AA3.73 & 0.1112 & 91 & 0.00264 & 17 & 1.46719 & 1.88673 & 10 & 0.282387 & 49 & 0.282358 & -1.8 & 1.7 & 1.29 & 598 & 11 \\
\hline AA3.75 & 0.0320 & 26 & 0.00083 & 5 & 1.46716 & 1.88656 & 14 & 0.281617 & 21 & 0.281585 & 2.1 & 0.8 & 2.20 & 1970 & 19 \\
\hline AA3_77 & 0.0318 & 37 & 0.00078 & 8 & 1.46716 & 1.88650 & 16 & 0.281386 & 22 & 0.281353 & -0.3 & 0.8 & 2.54 & 2221 & 16 \\
\hline AA3_78 & 0.0289 & 23 & 0.00074 & 4 & 1.46730 & 1.88662 & 13 & 0.281582 & 21 & 0.281549 & 8.2 & 0.7 & 2.12 & 2289 & 20 \\
\hline AA3_82 & 0.0360 & 31 & 0.00086 & 5 & 1.46715 & 1.88648 & 11 & 0.281249 & 21 & 0.281208 & 1.4 & 0.7 & 2.68 & 2515 & 9 \\
\hline AA3_85 & 0.0231 & 20 & 0.00060 & 4 & 1.46718 & 1.88657 & 12 & 0.281541 & 21 & 0.281517 & 2.4 & 0.7 & 2.28 & 2087 & 23 \\
\hline AA3_86 & 0.0445 & 51 & 0.00111 & 12 & 1.46718 & 1.88678 & 12 & 0.282202 & 28 & 0.282189 & -7.5 & 1.0 & 1.61 & 610 & 10 \\
\hline AA3_87 & 0.0231 & 25 & 0.00059 & 5 & 1.46730 & 1.88676 & 13 & 0.281557 & 22 & 0.281534 & -0.1 & 0.8 & 2.31 & 1955 & 18 \\
\hline AA3_88 & 0.0160 & 13 & 0.00040 & 3 & 1.46718 & 1.88655 & 12 & 0.281593 & 18 & 0.281580 & -2.1 & 0.6 & 2.29 & 1795 & 23 \\
\hline AA3_91 & 0.0471 & 55 & 0.00117 & 13 & 1.46736 & 1.88692 & 12 & 0.281386 & 24 & 0.281335 & 0.0 & 0.9 & 2.55 & 2261 & 13 \\
\hline AA3_93 & 0.1673 & 411 & 0.00299 & 67 & 1.46718 & 1.88648 & 12 & 0.281406 & 74 & 0.281266 & 2.0 & 2.6 & 2.60 & 2454 & 14 \\
\hline AA3_97 & 0.1173 & 97 & 0.00283 & 17 & 1.46731 & 1.88679 & 14 & 0.282277 & 53 & 0.282241 & -4.5 & 1.9 & 1.49 & 661 & 12 \\
\hline AA3_100 & 0.0344 & 34 & 0.00103 & 8 & 1.46714 & 1.88649 & 16 & 0.282553 & 25 & 0.282541 & 5.1 & 0.9 & 0.92 & 616 & 8 \\
\hline AA 3.110 & 0.0236 & 20 & 0.00069 & 4 & 1.46724 & 1.88680 & 15 & 0.281594 & 20 & 0.281566 & 5.9 & 0.7 & 2.15 & 2166 & 16 \\
\hline AA3_112 & 0.0733 & 61 & 0.00183 & 12 & 1.46718 & 1.88653 & 15 & 0.282289 & 32 & 0.282268 & -4.6 & 1.1 & 1.46 & 614 & 10 \\
\hline AA3_115 & 0.0147 & 13 & 0.00042 & 3 & 1.46713 & 1.88644 & 13 & 0.281640 & 20 & 0.281623 & 4.9 & 0.7 & 2.10 & 2031 & 16 \\
\hline AA 3.124 & 0.0672 & 55 & 0.00166 & 11 & 1.46725 & 1.88685 & 16 & 0.282289 & 32 & 0.282270 & -4.6 & 1.1 & 1.45 & 610 & 13 \\
\hline
\end{tabular}


Table 1 (Continued)

\begin{tabular}{|c|c|c|c|c|c|c|c|c|c|c|c|c|c|c|c|}
\hline & ${ }^{176} \mathrm{Yb} / /^{177} \mathrm{Hf}^{\mathrm{a}}$ & $\pm 2 \sigma$ & ${ }^{17} \mathrm{Lu}^{1 / 177} \mathrm{Hf}^{\mathrm{d}}$ & $\pm 2 \sigma$ & ${ }^{178} \mathrm{Hf} /{ }^{177} \mathrm{Hf}$ & ${ }^{180} \mathrm{Hf} /{ }^{177} \mathrm{Hf}$ & $\mathrm{Sig}_{\mathrm{Hf}}(\mathrm{V})$ & ${ }^{176} \mathrm{Hf} / /^{177} \mathrm{Hf}^{\mathrm{d}}$ & $\pm 2 \sigma^{c}$ & ${ }^{17}{ }^{6} \mathrm{Hf} / /^{177} \mathrm{Hf}_{(t)}$ & $\varepsilon H f(t)^{d}$ & $\pm 2 \sigma^{c}$ & $\mathrm{~T}_{\mathrm{M} 2}{ }^{e}(\mathrm{Ga})$ & $\operatorname{Age}^{\mathrm{f}}(\mathrm{Ma})$ & $\pm 2 \sigma$ \\
\hline AA3.125 & 0.0614 & 71 & 0.00165 & 14 & 1.46718 & 1.88664 & 17 & 0.281616 & 42 & 0.281559 & -3.0 & 1.5 & 2.33 & 1789 & 22 \\
\hline AA4_6 & 0.0160 & 15 & 0.00050 & 5 & 1.46736 & 1.88684 & 12 & 0.281438 & 17 & 0.281417 & 1.9 & 0.6 & 2.41 & 2218 & 21 \\
\hline AA4.7 & 0.0242 & 19 & 0.00083 & 6 & 1.46734 & 1.88692 & 21 & 0.281471 & 18 & 0.281441 & -4.1 & 0.6 & 2.50 & 1923 & 13 \\
\hline AA4_-9 & 0.0321 & 28 & 0.00093 & 9 & 1.46725 & 1.88675 & 17 & 0.281359 & 20 & 0.281320 & -1.8 & 0.7 & 2.61 & 2210 & 19 \\
\hline AA 4_ 10 & 0.0188 & 12 & 0.00057 & 4 & 1.46732 & 1.88691 & 13 & 0.281548 & 18 & 0.281527 & -0.7 & 0.7 & 2.33 & 1940 & 14 \\
\hline AA4_11 & 0.0075 & 5 & 0.00027 & 2 & 1.46734 & 1.88675 & 12 & 0.281548 & 20 & 0.281537 & 4.1 & 0.7 & 2.22 & 2130 & 27 \\
\hline AA 4_12 & 0.0431 & 35 & 0.00133 & 10 & 1.46736 & 1.88693 & 15 & 0.281609 & 27 & 0.281556 & 3.3 & 1.0 & 2.21 & 2066 & 24 \\
\hline AA4_13 & 0.0190 & 16 & 0.00055 & 4 & 1.46731 & 1.88681 & 15 & 0.281681 & 17 & 0.281662 & 1.3 & 0.6 & 2.12 & 1819 & 19 \\
\hline AA 4_ 16 & 0.0190 & 16 & 0.00051 & 4 & 1.46725 & 1.88669 & 15 & 0.281333 & 22 & 0.281309 & 3.4 & 0.8 & 2.52 & 2450 & 13 \\
\hline AA4_17 & 0.0354 & 38 & 0.00106 & 15 & 1.46727 & 1.88679 & 19 & 0.281576 & 23 & 0.281535 & 1.7 & 0.8 & 2.27 & 2032 & 18 \\
\hline AA4_19 & 0.0272 & 27 & 0.00080 & 9 & 1.46727 & 1.88693 & 16 & 0.282043 & 19 & 0.282034 & -12.8 & 0.7 & 1.91 & 616 & 10 \\
\hline AA 4_20 & 0.0286 & 21 & 0.00086 & 7 & 1.46730 & 1.88676 & 18 & 0.281592 & 19 & 0.281557 & 3.9 & 0.7 & 2.20 & 2088 & 11 \\
\hline AA4_21 & 0.0342 & 28 & 0.00092 & 9 & 1.46713 & 1.88648 & 15 & 0.281435 & 21 & 0.281394 & 3.9 & 0.8 & 2.40 & 2343 & 28 \\
\hline AA4223 & 0.0402 & 26 & 0.00109 & 7 & 1.46726 & 1.88663 & 13 & 0.281464 & 25 & 0.281418 & 1.4 & 0.9 & 2.42 & 2194 & 15 \\
\hline AA4_26 & 0.0095 & 8 & 0.00027 & 3 & 1.46729 & 1.88666 & 14 & 0.281324 & 19 & 0.281311 & 5.0 & 0.7 & 2.48 & 2516 & 16 \\
\hline AA4_29 & 0.0196 & 14 & 0.00065 & 5 & 1.46729 & 1.88664 & 13 & 0.282065 & 20 & 0.282058 & -11.8 & 0.7 & 1.86 & 624 & 23 \\
\hline AA4_30 & 0.0188 & 13 & 0.00064 & 5 & 1.46734 & 1.88694 & 10 & 0.281598 & 24 & 0.281574 & 1.2 & 0.9 & 2.23 & 1947 & 17 \\
\hline AA4_35 & 0.0028 & 9 & 0.00009 & 3 & 1.46731 & 1.88683 & 18 & 0.281527 & 19 & 0.281523 & 1.9 & 0.7 & 2.28 & 2055 & 51 \\
\hline AA4_36 & 0.0150 & 13 & 0.00059 & 6 & 1.46734 & 1.88687 & 15 & 0.281635 & 19 & 0.281613 & 2.2 & 0.7 & 2.16 & 1934 & 20 \\
\hline AA4_42 & 0.0140 & 10 & 0.00056 & 4 & 1.46736 & 1.88694 & 16 & 0.281629 & 20 & 0.281608 & 3.3 & 0.7 & 2.15 & 1986 & 12 \\
\hline AA4_43 & 0.0141 & 9 & 0.00041 & 3 & 1.46723 & 1.88655 & 13 & 0.281313 & 20 & 0.281294 & 3.1 & 0.7 & 2.54 & 2456 & 22 \\
\hline AA4-45b & 0.0306 & 19 & 0.00098 & 6 & 1.46730 & 1.88668 & 16 & 0.281660 & 23 & 0.281622 & 4.6 & 0.8 & 2.10 & 2021 & 17 \\
\hline AA 4.47 & 0.0257 & 59 & 0.00080 & 17 & 1.46726 & 1.88689 & 17 & 0.281592 & 20 & 0.281557 & 7.9 & 0.7 & 2.12 & 2264 & 34 \\
\hline AA4_48 & 0.0376 & 24 & 0.00109 & 7 & 1.46736 & 1.88691 & 17 & 0.281271 & 20 & 0.281221 & -0.4 & 0.7 & 2.70 & 2422 & 17 \\
\hline AA4_52 & 0.0242 & 21 & 0.00079 & 7 & 1.46735 & 1.88693 & 16 & 0.280998 & 21 & 0.280960 & -6.8 & 0.7 & 3.16 & 2542 & 16 \\
\hline AA4.59 & 0.0228 & 14 & 0.00059 & 4 & 1.46724 & 1.88666 & 17 & 0.281626 & 18 & 0.281604 & 4.4 & 0.6 & 2.13 & 2042 & 14 \\
\hline AA4_60 & 0.0292 & 26 & 0.00078 & 7 & 1.46718 & 1.88675 & 13 & 0.281180 & 20 & 0.281143 & -1.6 & 0.7 & 2.83 & 2487 & 18 \\
\hline AA4_66 & 0.0283 & 30 & 0.00085 & 9 & 1.46736 & 1.88694 & 13 & 0.281180 & 22 & 0.281143 & -6.8 & 0.8 & 2.93 & 2262 & 27 \\
\hline AA4_67 & 0.0016 & 4 & 0.00005 & 1 & 1.46726 & 1.88692 & 17 & 0.281410 & 16 & 0.281407 & 1.0 & 0.6 & 2.44 & 2197 & 43 \\
\hline AA4_68 & 0.0557 & 38 & 0.00171 & 11 & 1.46737 & 1.88694 & 16 & 0.281646 & 27 & 0.281577 & 5.3 & 0.9 & 2.15 & 2120 & 14 \\
\hline AA4_69 & 0.0354 & 48 & 0.00093 & 10 & 1.46724 & 1.88684 & 14 & 0.282573 & 23 & 0.282561 & 7.1 & 0.8 & 0.86 & 671 & 15 \\
\hline AA4.70 & 0.0159 & 10 & 0.00045 & 3 & 1.46723 & 1.88662 & 14 & 0.281581 & 18 & 0.281562 & 7.3 & 0.6 & 2.12 & 2228 & 32 \\
\hline AA4.75 & 0.0213 & 25 & 0.00056 & 6 & 1.46721 & 1.88656 & 14 & 0.281633 & 17 & 0.281611 & 4.8 & 0.6 & 2.11 & 2048 & 24 \\
\hline AA 4.76 & 0.0539 & 42 & 0.00162 & 14 & 1.46733 & 1.88692 & 12 & 0.281398 & 31 & 0.281330 & -1.8 & 1.1 & 2.60 & 2192 & 14 \\
\hline AA4_78 & 0.0172 & 14 & 0.00045 & 3 & 1.46721 & 1.88670 & 14 & 0.281608 & 17 & 0.281590 & 4.2 & 0.6 & 2.15 & 2052 & 17 \\
\hline AA4_80 & 0.0364 & 26 & 0.00105 & 7 & 1.46714 & 1.88646 & 14 & 0.281603 & 21 & 0.281564 & 1.1 & 0.7 & 2.25 & 1960 & 13 \\
\hline AA4_81 & 0.0162 & 11 & 0.00041 & 3 & 1.46715 & 1.88654 & 14 & 0.281134 & 17 & 0.281114 & -2.4 & 0.6 & 2.88 & 2497 & 16 \\
\hline AA4.83 & 0.0192 & 13 & 0.00050 & 3 & 1.46723 & 1.88678 & 15 & 0.281105 & 20 & 0.281084 & -9.0 & 0.7 & 3.05 & 2259 & 77 \\
\hline AA4_84 & 0.0250 & 15 & 0.00064 & 4 & 1.46722 & 1.88671 & 15 & 0.281349 & 19 & 0.281320 & 2.4 & 0.7 & 2.52 & 2389 & 11 \\
\hline AA4_86 & 0.0275 & 21 & 0.00071 & 5 & 1.46722 & 1.88655 & 14 & 0.281719 & 19 & 0.281695 & 3.1 & 0.7 & 2.04 & 1847 & 43 \\
\hline AA4_88 & 0.0124 & 10 & 0.00030 & 3 & 1.46723 & 1.88657 & 15 & 0.281513 & 18 & 0.281502 & -2.3 & 0.6 & 2.39 & 1908 & 17 \\
\hline AA4_90 & 0.0165 & 11 & 0.00047 & 3 & 1.46718 & 1.88659 & 15 & 0.281337 & 16 & 0.281315 & 3.6 & 0.6 & 2.51 & 2448 & 18 \\
\hline AA5_2 & 0.0295 & 22 & 0.00096 & 6 & 1.46721 & 1.88676 & 13 & 0.282680 & 24 & 0.282669 & 10.0 & 0.9 & 0.66 & 633 & 11 \\
\hline AA5_5 & 0.0276 & 25 & 0.00083 & 7 & 1.46717 & 1.88651 & 13 & 0.282582 & 18 & 0.282573 & 6.0 & 0.6 & 0.86 & 607 & 11 \\
\hline AA5_7 & 0.0238 & 18 & 0.00066 & 4 & 1.46718 & 1.88665 & 13 & 0.281612 & 22 & 0.281585 & 6.6 & 0.8 & 2.11 & 2164 & 69 \\
\hline AA5_8 & 0.0199 & 14 & 0.00058 & 5 & 1.46737 & 1.88692 & 18 & 0.281613 & 16 & 0.281593 & -1.1 & 0.6 & 2.25 & 1819 & 13 \\
\hline AA5 10 & 0.0496 & 34 & 0.00152 & 12 & 1.46724 & 1.88668 & 15 & 0.282521 & 27 & 0.282504 & 3.2 & 0.9 & 1.00 & 590 & 12 \\
\hline AA5_11 & 0.0198 & 21 & 0.00060 & 6 & 1.46734 & 1.88676 & 16 & 0.282459 & 19 & 0.282452 & 1.6 & 0.7 & 1.10 & 601 & 11 \\
\hline AA5.17 & 0.0242 & 33 & 0.00086 & 11 & 1.46736 & 1.88692 & 14 & 0.281593 & 20 & 0.281556 & 6.8 & 0.7 & 2.14 & 2215 & 24 \\
\hline AA5_18 & 0.0225 & 14 & 0.00060 & 4 & 1.46721 & 1.88675 & 15 & 0.281420 & 19 & 0.281395 & -1.2 & 0.7 & 2.50 & 2119 & 22 \\
\hline AA5.19 & 0.0449 & 31 & 0.00145 & 9 & 1.46726 & 1.88669 & 15 & 0.282583 & 28 & 0.282567 & 5.4 & 1.0 & 0.88 & 588 & 10 \\
\hline AA5_24 & 0.0744 & 67 & 0.00198 & 16 & 1.46716 & 1.88644 & 13 & 0.282611 & 34 & 0.282590 & 6.1 & 1.2 & 0.84 & 582 & 12 \\
\hline AA5_26 & 0.0156 & 11 & 0.00050 & 3 & 1.46722 & 1.88664 & 18 & 0.282665 & 18 & 0.282658 & 11.1 & 0.6 & 0.66 & 695 & 14 \\
\hline AA5_32 & 0.0207 & 28 & 0.00063 & 6 & 1.46717 & 1.88654 & 16 & 0.282658 & 18 & 0.282651 & 9.2 & 0.6 & 0.70 & 622 & 13 \\
\hline AA5_34 & 0.0207 & 20 & 0.00069 & 5 & 1.46727 & 1.88659 & 21 & 0.282366 & 15 & 0.282357 & -0.9 & 0.5 & 1.27 & 640 & 12 \\
\hline
\end{tabular}


Table 1 (Continued)

\begin{tabular}{|c|c|c|c|c|c|c|c|c|c|c|c|c|c|c|c|}
\hline & ${ }^{17} 6 \mathrm{Yb} / /^{177} \mathrm{Hf}^{\mathrm{a}}$ & $\pm 2 \sigma$ & ${ }^{17} \cdot \mathrm{Lu} /{ }^{177} \mathrm{Hf}^{\mathrm{a}}$ & $\pm 2 \sigma$ & ${ }^{178} \mathrm{Hf} /{ }^{177} \mathrm{Hf}$ & ${ }^{180} \mathrm{Hf} /{ }^{177} \mathrm{Hf}$ & $\mathrm{Sig}_{\mathrm{Hf}}(\mathrm{V})$ & ${ }^{17} \cdot \mathrm{Hf} / /^{177} \mathrm{Hf}^{\mathrm{d}}$ & $\pm 2 \sigma^{c}$ & ${ }^{17} \mathbf{6} \mathrm{Hf} / /^{177} \mathrm{Hf}_{(\mathrm{t})}$ & $\varepsilon H f(t)^{d}$ & $\pm 2 \sigma^{c}$ & $\mathrm{~T}_{\mathrm{MM} 2}{ }^{e}(\mathrm{Ga})$ & $\operatorname{Age}^{f}(\mathrm{Ma})$ & $\pm 2 \sigma$ \\
\hline AA5_37 & 0.0083 & 10 & 0.00023 & 2 & 1.46721 & 1.88645 & 16 & 0.282611 & 16 & 0.282608 & 7.9 & 0.6 & 0.78 & 632 & 13 \\
\hline AA5_38 & 0.0101 & 7 & 0.00031 & 2 & 1.46724 & 1.88664 & 15 & 0.282674 & 20 & 0.282670 & 9.4 & 0.7 & 0.67 & 602 & 11 \\
\hline AA5_42 & 0.0331 & 25 & 0.00097 & 7 & 1.46722 & 1.88654 & 17 & 0.282637 & 20 & 0.282626 & 8.7 & 0.7 & 0.74 & 640 & 15 \\
\hline AA5_43 & 0.0178 & 18 & 0.00057 & 4 & 1.46721 & 1.88665 & 14 & 0.282647 & 18 & 0.282639 & 10.2 & 0.7 & 0.70 & 685 & 15 \\
\hline AA5_44 & 0.0226 & 20 & 0.00070 & 5 & 1.46722 & 1.88668 & 17 & 0.282571 & 18 & 0.282563 & 6.7 & 0.7 & 0.86 & 650 & 13 \\
\hline AA5_45 & 0.0323 & 29 & 0.00111 & 8 & 1.46735 & 1.88690 & 18 & 0.282580 & 20 & 0.282567 & 6.1 & 0.7 & 0.87 & 619 & 12 \\
\hline AA5_46 & 0.0263 & 26 & 0.00079 & 7 & 1.46723 & 1.88650 & 16 & 0.282043 & 20 & 0.282034 & -12.8 & 0.7 & 1.91 & 616 & 11 \\
\hline AA5_47 & 0.0194 & 13 & 0.00059 & 4 & 1.46713 & 1.88653 & 17 & 0.282682 & 17 & 0.282675 & 11.5 & 0.6 & 0.63 & 690 & 16 \\
\hline AA5_49 & 0.0130 & 9 & 0.00038 & 2 & 1.46736 & 1.88690 & 15 & 0.282662 & 17 & 0.282657 & 9.1 & 0.6 & 0.70 & 608 & 12 \\
\hline AA5_52 & 0.0316 & 20 & 0.00084 & 5 & 1.46718 & 1.88654 & 19 & 0.282679 & 19 & 0.282669 & 9.9 & 0.7 & 0.66 & 627 & 11 \\
\hline AA5_54 & 0.0261 & 22 & 0.00098 & 10 & 1.46728 & 1.88669 & 18 & 0.282390 & 20 & 0.282378 & 0.2 & 0.7 & 1.22 & 655 & 13 \\
\hline AA5_57 & 0.0725 & 106 & 0.00187 & 27 & 1.46738 & 1.88692 & 13 & 0.281618 & 31 & 0.281545 & 2.5 & 1.1 & 2.24 & 2052 & 13 \\
\hline AA5_62 & 0.0551 & 52 & 0.00162 & 15 & 1.46720 & 1.88651 & 10 & 0.282674 & 31 & 0.282653 & 11.0 & 1.1 & 0.66 & 699 & 14 \\
\hline AA5_64 & 0.0241 & 21 & 0.00077 & 6 & 1.46721 & 1.88650 & 10 & 0.282512 & 28 & 0.282503 & 4.3 & 1.0 & 0.99 & 638 & 13 \\
\hline AA5_66b & 0.0179 & 20 & 0.00053 & 4 & 1.46715 & 1.88648 & 12 & 0.282012 & 20 & 0.282005 & -13.9 & 0.7 & 1.96 & 613 & 15 \\
\hline AA5_69 & 0.0337 & 27 & 0.00127 & 10 & 1.46714 & 1.88656 & 6 & 0.281447 & 29 & 0.281393 & 1.3 & 1.0 & 2.46 & 2229 & 19 \\
\hline AA5_74 & 0.0189 & 14 & 0.00067 & 5 & 1.46730 & 1.88691 & 14 & 0.282576 & 19 & 0.282568 & 7.6 & 0.7 & 0.84 & 685 & 14 \\
\hline AA5_76 & 0.0455 & 30 & 0.00123 & 8 & 1.46727 & 1.88668 & 11 & 0.282439 & 26 & 0.282425 & 0.3 & 0.9 & 1.16 & 583 & 14 \\
\hline AA5_81 & 0.0128 & 10 & 0.00037 & 3 & 1.46721 & 1.88670 & 16 & 0.282677 & 19 & 0.282672 & 11.0 & 0.7 & 0.64 & 671 & 13 \\
\hline AA5_88 & 0.0218 & 22 & 0.00063 & 7 & 1.46722 & 1.88666 & 12 & 0.281188 & 19 & 0.281157 & 1.8 & 0.7 & 2.74 & 2610 & 14 \\
\hline AA5_90 & 0.0425 & 30 & 0.00126 & 9 & 1.46728 & 1.88677 & 12 & 0.282523 & 22 & 0.282509 & 3.4 & 0.8 & 0.99 & 591 & 14 \\
\hline AA5_98 & 0.0214 & 15 & 0.00067 & 4 & 1.46715 & 1.88663 & 15 & 0.282642 & 18 & 0.282635 & 7.9 & 0.6 & 0.75 & 593 & 10 \\
\hline AA5_102 & 0.0201 & 36 & 0.00062 & 11 & 1.46718 & 1.88667 & 12 & 0.282641 & 21 & 0.282634 & 7.7 & 0.7 & 0.75 & 583 & 19 \\
\hline AA5_107 & 0.0203 & 24 & 0.00065 & 6 & 1.46713 & 1.88644 & 14 & 0.282048 & 22 & 0.282041 & -12.6 & 0.8 & 1.90 & 617 & 11 \\
\hline AA5_109 & 0.0241 & 15 & 0.00074 & 5 & 1.46716 & 1.88649 & 16 & 0.282579 & 16 & 0.282570 & 6.4 & 0.6 & 0.86 & 627 & 13 \\
\hline AA5_112 & 0.0513 & 43 & 0.00129 & 9 & 1.46726 & 1.88677 & 16 & 0.282402 & 22 & 0.282386 & 0.2 & 0.8 & 1.21 & 641 & 13 \\
\hline AA5_115 & 0.0167 & 22 & 0.00046 & 6 & 1.46714 & 1.88645 & 15 & 0.282693 & 19 & 0.282686 & 13.8 & 0.7 & 0.57 & 773 & 18 \\
\hline AA5_117 & 0.0260 & 22 & 0.00068 & 6 & 1.46721 & 1.88656 & 14 & 0.282601 & 17 & 0.282593 & 7.3 & 0.6 & 0.81 & 630 & 13 \\
\hline AA5_118 & 0.0232 & 20 & 0.00061 & 5 & 1.46712 & 1.88657 & 14 & 0.282186 & 21 & 0.282179 & -7.3 & 0.7 & 1.62 & 633 & 12 \\
\hline AA5_121 & 0.0324 & 23 & 0.00099 & 6 & 1.46712 & 1.88645 & 14 & 0.282596 & 23 & 0.282584 & 7.2 & 0.8 & 0.83 & 638 & 12 \\
\hline AA5_122 & 0.0339 & 26 & 0.00090 & 7 & $\begin{array}{l}1.40712 \\
1.46717\end{array}$ & 1.88644 & 16 & 0.282693 & 19 & 0.282682 & 10.4 & 0.7 & 0.64 & 629 & 13 \\
\hline AA5_123 & 0.0095 & 10 & 0.00029 & 3 & 1.46713 & 1.88646 & 17 & 0.282672 & 14 & 0.282669 & 10.0 & 0.5 & 0.66 & 633 & 14 \\
\hline AA6_1 & 0.0377 & 48 & 0.00109 & 15 & 1.46712 & 1.88647 & 15 & 0.281586 & 27 & 0.281543 & 3.2 & 0.9 & 2.23 & 2084 & 15 \\
\hline AA6_4 & 0.0345 & 21 & 0.00103 & 6 & 1.46722 & 1.88665 & 13 & 0.282130 & 25 & 0.282117 & -9.3 & 0.9 & 1.74 & 643 & 13 \\
\hline AA6_7 & 0.0318 & 20 & 0.00098 & 6 & 1.46722 & 1.88659 & 13 & 0.281596 & 23 & 0.281558 & 3.4 & 0.8 & 2.21 & 2068 & 14 \\
\hline AA6_12 & 0.0807 & 65 & 0.00228 & 18 & 1.46719 & 1.88678 & 12 & 0.282024 & 32 & 0.281998 & -14.2 & 1.1 & 1.98 & 612 & 8 \\
\hline AA6_13 & 0.0256 & 24 & 0.00087 & 9 & 1.46713 & 1.88645 & 9 & 0.281606 & 25 & 0.281570 & 6.2 & 0.9 & 2.13 & 2169 & 16 \\
\hline AA6_15 & 0.0353 & 27 & 0.00097 & 7 & 1.46736 & 1.88693 & 11 & 0.281473 & 25 & 0.281434 & 0.9 & 0.9 & 2.41 & 2152 & 11 \\
\hline AA6_16 & 0.0341 & 39 & 0.00101 & 9 & 1.46717 & 1.88652 & 12 & 0.281582 & 24 & 0.281542 & 3.3 & 0.9 & 2.23 & 2087 & 16 \\
\hline AA6_23 & 0.0154 & 24 & 0.00046 & 9 & 1.46723 & 1.88648 & 12 & 0.281641 & 23 & 0.281623 & 6.8 & 0.8 & 2.06 & 2114 & 7 \\
\hline AA6_29 & 0.0310 & 23 & 0.00082 & 6 & 1.46717 & 1.88666 & 12 & 0.281611 & 22 & 0.281577 & 7.1 & 0.8 & 2.11 & 2198 & 24 \\
\hline AA6_34 & 0.0317 & 56 & 0.00084 & 15 & 1.46729 & 1.88667 & 14 & 0.281581 & 22 & 0.281546 & 6.7 & 0.8 & 2.16 & 2227 & 11 \\
\hline AA6_35 & 0.0265 & 18 & 0.00075 & 5 & 1.46723 & 1.88667 & 10 & 0.282015 & 24 & 0.282007 & -14.0 & 0.9 & 1.96 & 609 & 10 \\
\hline AA6_-42 & 0.0448 & 40 & 0.00123 & 10 & 1.46715 & 1.88646 & 13 & 0.282029 & 25 & 0.282015 & -13.5 & 0.9 & 1.94 & 619 & 10 \\
\hline AA6_46 & 0.0100 & 13 & 0.00025 & 3 & 1.46728 & 1.88668 & 13 & 0.281582 & 19 & 0.281573 & 3.4 & 0.7 & 2.19 & 2046 & 10 \\
\hline AA6_-52 & 0.0287 & 20 & 0.00081 & 6 & 1.46718 & 1.88668 & 11 & 0.281619 & 21 & 0.281586 & 5.5 & 0.7 & 2.13 & 2114 & 13 \\
\hline AA6_52a & 0.0287 & 20 & 0.00081 & 6 & 1.46718 & 1.88668 & 11 & 0.281619 & 21 & 0.281586 & 5.5 & 0.7 & 2.13 & 2114 & 13 \\
\hline AA6_83b & 0.0329 & 57 & 0.00076 & 13 & 1.46730 & 1.88682 & 15 & 0.281601 & 24 & 0.281572 & 3.2 & 0.9 & 2.19 & 2039 & 13 \\
\hline AA6_84 & 0.0755 & 158 & 0.00162 & 33 & 1.46725 & 1.88661 & 16 & 0.281598 & 35 & 0.281536 & 1.3 & 1.2 & 2.28 & 2012 & 17 \\
\hline AA6_85 & 0.0214 & 20 & 0.00058 & 5 & 1.46724 & 1.88675 & 13 & 0.281641 & 22 & 0.281619 & 4.6 & 0.8 & 2.11 & 2028 & 16 \\
\hline AA6_87 & 0.0392 & 33 & 0.00089 & 8 & 1.46733 & 1.88684 & 17 & 0.281604 & 23 & 0.281569 & 3.4 & 0.8 & 2.19 & 2054 & 14 \\
\hline AA6_88 & 0.0644 & 42 & 0.00173 & 11 & 1.46727 & 1.88674 & 13 & 0.281615 & 28 & 0.281548 & 2.3 & 1.0 & 2.24 & 2036 & 23 \\
\hline AA6_90 & 0.0419 & 51 & 0.00105 & 12 & 1.46732 & 1.88693 & 12 & 0.281602 & 28 & 0.281561 & 3.2 & 1.0 & 2.21 & 2054 & 13 \\
\hline AA6_92 & 0.0641 & 52 & 0.00167 & 13 & 1.46733 & 1.88694 & 11 & 0.281590 & 28 & 0.281525 & 1.9 & 1.0 & 2.28 & 2054 & 21 \\
\hline AA6_93 & 0.0742 & 85 & 0.00199 & 22 & 1.46721 & 1.88665 & 11 & 0.281622 & 37 & 0.281544 & 2.9 & 1.3 & 2.24 & 2068 & 13 \\
\hline AA6_96 & 0.0276 & 23 & 0.00076 & 6 & 1.46732 & 1.88674 & 14 & 0.281388 & 29 & 0.281357 & -1.2 & 1.0 & 2.55 & 2180 & 11 \\
\hline AA6_97 & 0.0194 & 46 & 0.00042 & 10 & 1.46728 & 1.88668 & 15 & 0.281574 & 21 & 0.281557 & 4.5 & 0.7 & 2.19 & 2118 & 15 \\
\hline
\end{tabular}


Table 1 (Continued)

\begin{tabular}{|c|c|c|c|c|c|c|c|c|c|c|c|c|c|c|c|}
\hline & ${ }^{17} 6 \mathrm{Yb} /{ }^{177} \mathrm{Hf}^{\mathrm{a}}$ & $\pm 2 \sigma$ & ${ }^{17}{ }^{17} \mathrm{Lu} /{ }^{177} \mathrm{Hf}^{\mathrm{a}}$ & $\pm 2 \sigma$ & ${ }^{178} \mathrm{Hf} /{ }^{177} \mathrm{Hf}$ & ${ }^{180} \mathrm{Hf} /{ }^{177} \mathrm{Hf}$ & $\mathrm{Sig}_{\mathrm{Hf}}(\mathrm{V})$ & ${ }^{17}{ }^{6} \mathrm{Hf} / /^{177} \mathrm{Hf}^{\mathrm{d}}$ & $\pm 2 \sigma^{c}$ & ${ }^{17}{ }^{6} \mathrm{Hf} / /^{177} \mathrm{Hf}_{(\mathrm{t})}$ & $\varepsilon H f(t)^{d}$ & $\pm 2 \sigma^{c}$ & $\mathrm{~T}_{\mathrm{M} 2}{ }^{e}(\mathrm{Ga})$ & $\operatorname{Age}^{f}(\mathrm{Ma})$ & \pm 20 \\
\hline AA6_ 100 & 0.0418 & 44 & 0.00114 & 13 & 1.46712 & 1.88644 & 13 & 0.281605 & 24 & 0.281561 & 2.9 & 0.8 & 2.21 & 2044 & 16 \\
\hline AA6. 103 & 0.0492 & 70 & 0.00122 & 16 & 1.46727 & 1.88668 & 15 & 0.281575 & 26 & 0.281527 & 2.9 & 0.9 & 2.26 & 2095 & 13 \\
\hline AA6. 105 & 0.0297 & 26 & 0.00073 & 6 & 1.46732 & 1.88674 & 14 & 0.281580 & 22 & 0.281552 & 3.4 & 0.8 & 2.22 & 2076 & 40 \\
\hline AA6- 108 & 0.0174 & 27 & 0.00040 & 7 & 1.46727 & 1.88658 & 15 & 0.281575 & 19 & 0.281559 & 4.6 & 0.7 & 2.18 & 2117 & 23 \\
\hline AA6_ 111 & 0.0538 & 54 & 0.00143 & 15 & 1.46723 & 1.88652 & 13 & 0.281636 & 26 & 0.281580 & 3.5 & 0.9 & 2.18 & 2039 & 18 \\
\hline AA7_-2 & 0.0364 & 25 & 0.00088 & 6 & 1.46716 & 1.88650 & 13 & 0.282567 & 20 & 0.282557 & 4.8 & 0.7 & 0.91 & 574 & 15 \\
\hline AA7-6 & 0.0330 & 28 & 0.00077 & 6 & 1.46724 & 1.88661 & 14 & 0.282524 & 20 & 0.282516 & 2.8 & 0.7 & 1.00 & 552 & 15 \\
\hline AA7_ 10 & 0.0313 & 21 & 0.00077 & 5 & 1.46716 & 1.88647 & 15 & 0.282516 & 18 & 0.282508 & 2.7 & 0.7 & 1.01 & 558 & 13 \\
\hline AA7- 12 & 0.0425 & 28 & 0.00106 & 7 & 1.46729 & 1.88679 & 12 & 0.282573 & 26 & 0.282562 & 5.3 & 0.9 & 0.89 & 592 & 14 \\
\hline AA7_ 16 & 0.0369 & 30 & 0.00086 & 7 & 1.46717 & 1.88650 & 15 & 0.282523 & 25 & 0.282514 & 2.6 & 0.9 & 1.00 & 546 & 14 \\
\hline AA7_ 18 & 0.0416 & 31 & 0.00100 & 7 & 1.46722 & 1.88661 & 13 & 0.282532 & 24 & 0.282522 & 2.8 & 0.9 & 0.99 & 543 & 13 \\
\hline AA7-20 & 0.0248 & 15 & 0.00062 & 4 & 1.46713 & 1.88644 & 10 & 0.282526 & 21 & 0.282520 & 2.9 & 0.8 & 0.99 & 550 & 14 \\
\hline AA7-23 & 0.0428 & 29 & 0.00100 & 7 & 1.46717 & 1.88648 & 16 & 0.282546 & 23 & 0.282535 & 3.8 & 0.8 & 0.95 & 567 & 11 \\
\hline AA7-24 & 0.0464 & 74 & 0.00109 & 14 & 1.46722 & 1.88662 & 15 & 0.282511 & 23 & 0.282499 & 2.7 & 0.8 & 1.02 & 574 & 15 \\
\hline AA7-28 & 0.0703 & 55 & 0.00168 & 12 & 1.46714 & 1.88647 & 18 & 0.282605 & 37 & 0.282586 & 6.3 & 1.3 & 0.84 & 599 & 10 \\
\hline AA7.29 & 0.0542 & 42 & 0.00125 & 8 & 1.46720 & 1.88682 & 18 & 0.282510 & 26 & 0.282497 & 2.8 & 0.9 & 1.02 & 584 & 11 \\
\hline AA7-30 & 0.0672 & 43 & 0.00164 & 11 & 1.46713 & 1.88646 & 13 & 0.282556 & 30 & 0.282539 & 3.7 & 1.1 & 0.95 & 556 & 10 \\
\hline AA7_31 & 0.0826 & 190 & 0.00194 & 36 & 1.46715 & 1.88644 & 12 & 0.282520 & 36 & 0.282499 & 2.8 & 1.3 & 1.02 & 581 & 12 \\
\hline AA7_32 & 0.0297 & 24 & 0.00075 & 6 & 1.46720 & 1.88651 & 12 & 0.282528 & 26 & 0.282520 & 3.5 & 0.9 & 0.98 & 579 & 13 \\
\hline AA7_34 & 0.0371 & 23 & 0.00092 & 6 & 1.46713 & 1.88654 & 15 & 0.282518 & 18 & 0.282508 & 2.8 & 0.6 & 1.01 & 563 & 10 \\
\hline AA7_35 & 0.0871 & 65 & 0.00205 & 16 & 1.46719 & 1.88651 & 12 & 0.282579 & 37 & 0.282557 & 4.7 & 1.3 & 0.91 & 570 & 11 \\
\hline AA7.36 & 0.0528 & 40 & 0.00124 & 8 & 1.46721 & 1.88659 & 19 & 0.282499 & 22 & 0.282485 & 2.7 & 0.8 & 1.04 & 596 & 11 \\
\hline AA7_37 & 0.0343 & 21 & 0.00086 & 5 & 1.46723 & 1.88657 & 14 & 0.282504 & 17 & 0.282495 & 2.9 & 0.6 & 1.02 & 590 & 10 \\
\hline AA7_40 & 0.0529 & 57 & 0.00122 & 12 & 1.46716 & 1.88668 & 16 & 0.282644 & 26 & 0.282629 & 8.4 & 0.9 & 0.75 & 621 & 12 \\
\hline AA7_42 & 0.0803 & 93 & 0.00159 & 15 & 1.46719 & 1.88674 & 18 & 0.282571 & 37 & 0.282553 & 4.7 & 1.3 & 0.91 & 579 & 12 \\
\hline AA7-43 & 0.0616 & 46 & 0.00145 & 10 & 1.46725 & 1.88667 & 15 & 0.282551 & 26 & 0.282536 & 3.8 & 0.9 & 0.95 & 564 & 10 \\
\hline AA7_46 & 0.0923 & 128 & 0.00222 & 27 & 1.46717 & 1.88646 & 12 & 0.282488 & 37 & 0.282465 & 1.2 & 1.3 & 1.09 & 563 & 10 \\
\hline AA7_47 & 0.0399 & 45 & 0.00090 & 11 & 1.46718 & 1.88656 & 18 & 0.281642 & 31 & 0.281607 & 4.4 & 1.1 & 2.13 & 2037 & 15 \\
\hline AA7-48 & 0.0336 & 24 & 0.00083 & 6 & 1.46731 & 1.88691 & 11 & 0.282538 & 20 & 0.282529 & 3.5 & 0.7 & 0.97 & 561 & 11 \\
\hline AA7_49 & 0.0440 & 27 & 0.00109 & 8 & 1.46714 & 1.88650 & 12 & 0.282523 & 26 & 0.282512 & 2.6 & 0.9 & 1.01 & 552 & 10 \\
\hline AA7.52 & 0.0287 & 26 & 0.00071 & 6 & 1.46722 & 1.88657 & 14 & 0.282529 & 21 & 0.282521 & 3.1 & 0.7 & 0.99 & 556 & 11 \\
\hline AA7.53 & 0.0607 & 80 & 0.00145 & 16 & 1.46716 & 1.88670 & 13 & 0.282490 & 27 & 0.282474 & 2.1 & 1.0 & 1.07 & 586 & 12 \\
\hline AA7_56 & 0.0735 & 45 & 0.00156 & 10 & 1.46714 & 1.88661 & 15 & 0.282537 & 28 & 0.282521 & 3.1 & 1.0 & 0.99 & 557 & 11 \\
\hline AA7_57 & 0.0701 & 52 & 0.00148 & 11 & 1.46713 & 1.88646 & 15 & 0.282563 & 27 & 0.282548 & 4.0 & 1.0 & 0.93 & 555 & 10 \\
\hline AA7.59 & 0.0530 & 35 & 0.00129 & 8 & 1.46726 & 1.88670 & 11 & 0.282540 & 24 & 0.282527 & 3.2 & 0.8 & 0.98 & 554 & 10 \\
\hline AA7_60 & 0.0325 & 22 & 0.00078 & 5 & 1.46724 & 1.88670 & 13 & 0.282253 & 42 & 0.282245 & -6.6 & 1.5 & 1.52 & 560 & 16 \\
\hline AA7-60 & 0.0397 & 50 & 0.00098 & 14 & 1.46716 & 1.88657 & 15 & 0.281641 & 62 & 0.281601 & 6.1 & 2.2 & 2.10 & 2121 & 17 \\
\hline AA7_61 & 0.2873 & 448 & 0.00495 & 74 & 1.46715 & 1.88646 & 14 & 0.282630 & 100 & 0.282576 & 5.7 & 3.5 & 0.87 & 585 & 11 \\
\hline AA7_63 & 0.0485 & 39 & 0.00103 & 8 & 1.46724 & 1.88686 & 15 & 0.282509 & 22 & 0.282498 & 2.8 & 0.8 & 1.02 & 580 & 12 \\
\hline AA7-64 & 0.0507 & 49 & 0.00126 & 13 & 1.46721 & 1.88653 & 13 & 0.282548 & 25 & 0.282535 & 3.2 & 0.9 & 0.96 & 540 & 11 \\
\hline AA7_68 & 0.0193 & 14 & 0.00051 & 3 & 1.46712 & 1.88647 & 11 & 0.282515 & 19 & 0.282510 & 2.7 & 0.7 & 1.01 & 556 & 13 \\
\hline AA7-70 & 0.0404 & 26 & 0.00102 & 6 & 1.46725 & 1.88662 & 15 & 0.282506 & 19 & 0.282495 & 2.0 & 0.7 & 1.04 & 548 & 12 \\
\hline AA7.73 & 0.0357 & 22 & 0.00091 & 6 & 1.46718 & 1.88657 & 14 & 0.282508 & 20 & 0.282498 & 2.0 & 0.7 & 1.04 & 544 & 10 \\
\hline AA7.75 & 0.0620 & 66 & 0.00157 & 18 & 1.46723 & 1.88657 & 12 & 0.282531 & 25 & 0.282515 & 2.6 & 0.9 & 1.00 & 543 & 10 \\
\hline JMC $475, n=14$ & & - & - & - & 1.46716 & 1.88667 & 19 & 0.282146 & 9 & & & & & & \\
\hline $\mathrm{GJ}-1, n=23$ & 0.0100 & 16 & 0.00031 & 2 & 1.46723 & 1.88666 & 11 & 0.282006 & 22 & 0.282003 & -13.9 & 0.8 & 2.18 & 606 & 4 \\
\hline Ples., $n=18$ & 0.0072 & 19 & 0.00019 & 6 & 1.46722 & 1.88666 & 22 & 0.282489 & 16 & 0.282489 & -2.6 & 0.6 & 1.35 & 338 & 2 \\
\hline
\end{tabular}

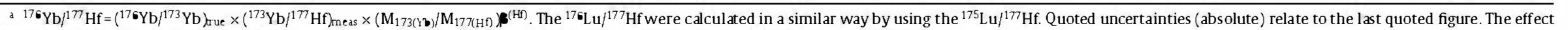
of the inter-element fractionation on the Lu/Hf was estimated to be about $6 \%$ or less based on analyses of the GJ-1 and Plesovice zircons.

- Mean Hf signal in volt.

c Uncertainties are quadratic additions of the within-run error and the daily reproducibility of the $40 \mathrm{ppb}$ JMC 475 solution. Uncertainties for theJMC475, GJ-1 and Plesovice are 2SD

d Initial ${ }^{17} \cdot \mathrm{Hf} /{ }^{177} \mathrm{Hf}$ and $\varepsilon \mathrm{Hf}$ calculated using the age determined by lA-ICP-MS dating (see last two rows).

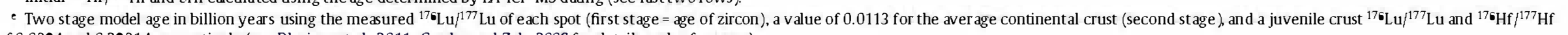
of 0.0384 and 0.28314 , respectively (see Dhuime et al., 2011; Gerdes and Zeh, 2006 for details and references).

${ }^{\mathrm{f}} \mathrm{U}-\mathrm{Pb} \mathrm{lA}-\mathrm{ICP}-\mathrm{MS}$ age and error. 


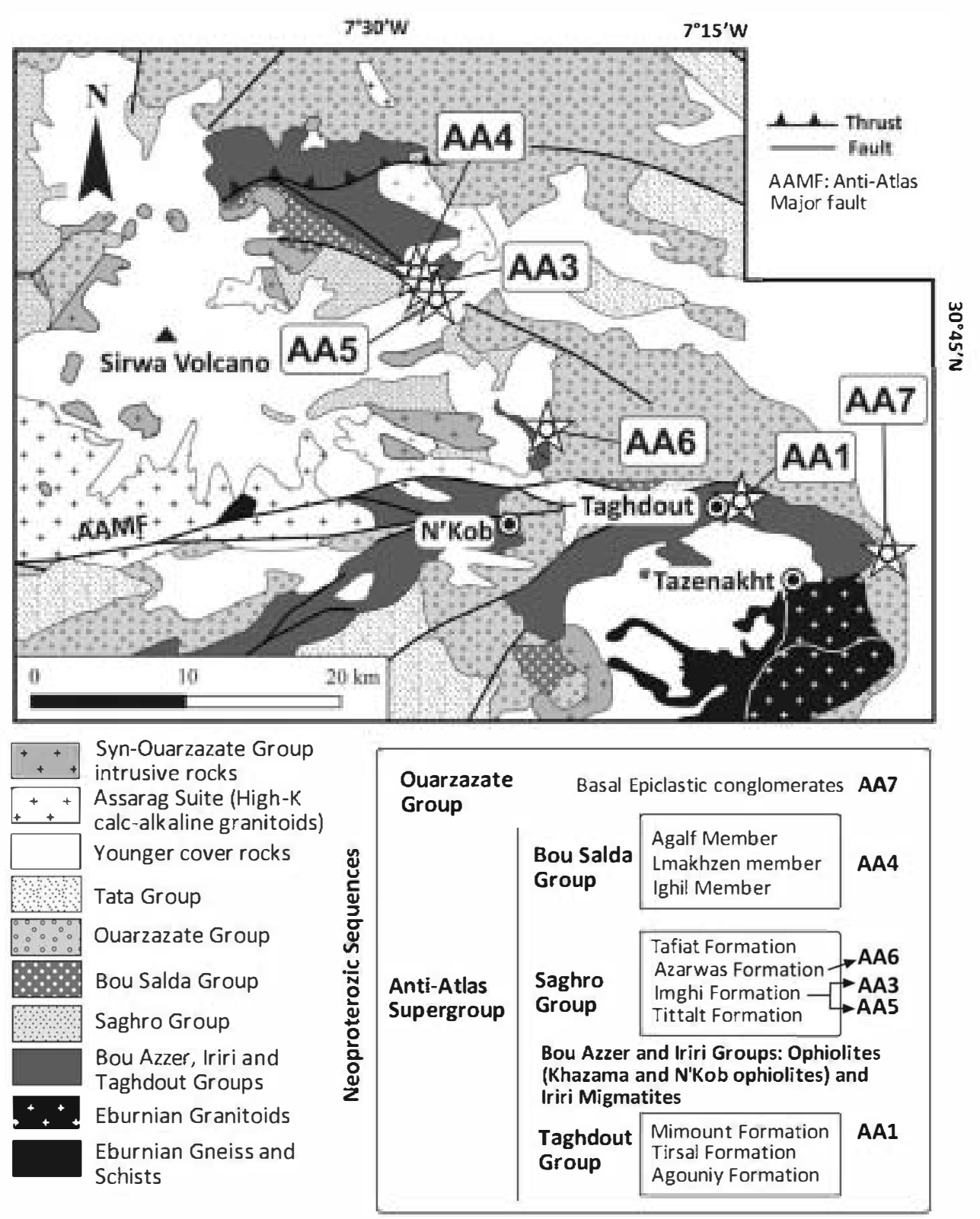

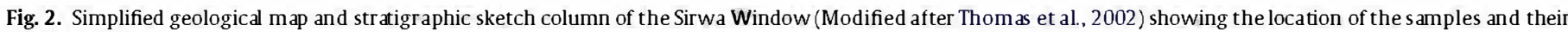
relative position.

group with positive $\varepsilon \mathrm{Hf}_{\mathrm{i}}$ forms a rough linear array nearly parallel to the ${ }^{176} \mathrm{Lu} /{ }^{177} \mathrm{Hf}$ evolution of a continental crust extracted from the depleted mantle during the Pan-African cycle (Fig. 3b). Zircons with values closets to the depleted mantle array have ages between 620 and $720 \mathrm{Ma}$, consistent with the formation of juvenile crust in an early island-arc setting. Zircon with ages of $600-550$ have somewhat lower $\boldsymbol{\varepsilon} \mathrm{Hf}_{\mathrm{i}}$ suggesting that juvenile magmas where contaminated by older crust or recycling of the juvenile island arcs together with some older crust. Both scenarios are compatible with a continental arc setting. Negative $\varepsilon \mathrm{Hf}_{\mathrm{i}}$ values occur mainly in the interval 590-650 Ma. A group of 10 zircons with the lowest negative $\varepsilon \mathrm{Hf}_{\mathrm{i}}$, between -10 and -15 and $T_{\mathrm{DM}}$ model ages between 1.85 and $2.0 \mathrm{Ga}$ can be explained by almost pure reworking of older Eburnian crust, while the relatively numerous group with negative intermediate values are only compatible with mixing of the Eburnian and the newly formed juvenile crust (Fig. 3a and b). As the formation of juvenile crust is the main magmatic process expected in an arc environment, the predominance of a juvenile Hf isotope signature suggests an island arc and a continental arc as most likely origin for the magmas from which the detrital zircons derived from. Although Neoproterozoic zircons from the Anti-Atlas Supergroup form a coherent group (Fig. 3b) some differences related to stratigraphic position can be highlighted. Samples AA3 and AA5 have different $\varepsilon \mathrm{Hf}$, with a tendency to positive values in AA5 and negative in AA3, despite they belong to the same formation (Fig. 2). This fact can be probably explained because AA3 is a diamictite, with a potential for containing ice-rafted debris which could be far-traveled.

Zircons of Neoproterozoic age are present in all the samples, except in the oldest passive margin Taghdout quartzite (AA1). Going to the top of the sedimentary sequence, the proportion of Neoproterozoic zircons in each sample is $80 \%$ (AA5), $52 \%$ (AA3), $7 \%$ (AA6), $\mathbf{5 \%}$ (AA4) and $\mathbf{9 7 \%}$ (AA7), being the youngest zircon population 610-620 Ma. Abati et al. (2010) proposed that this young zircon 

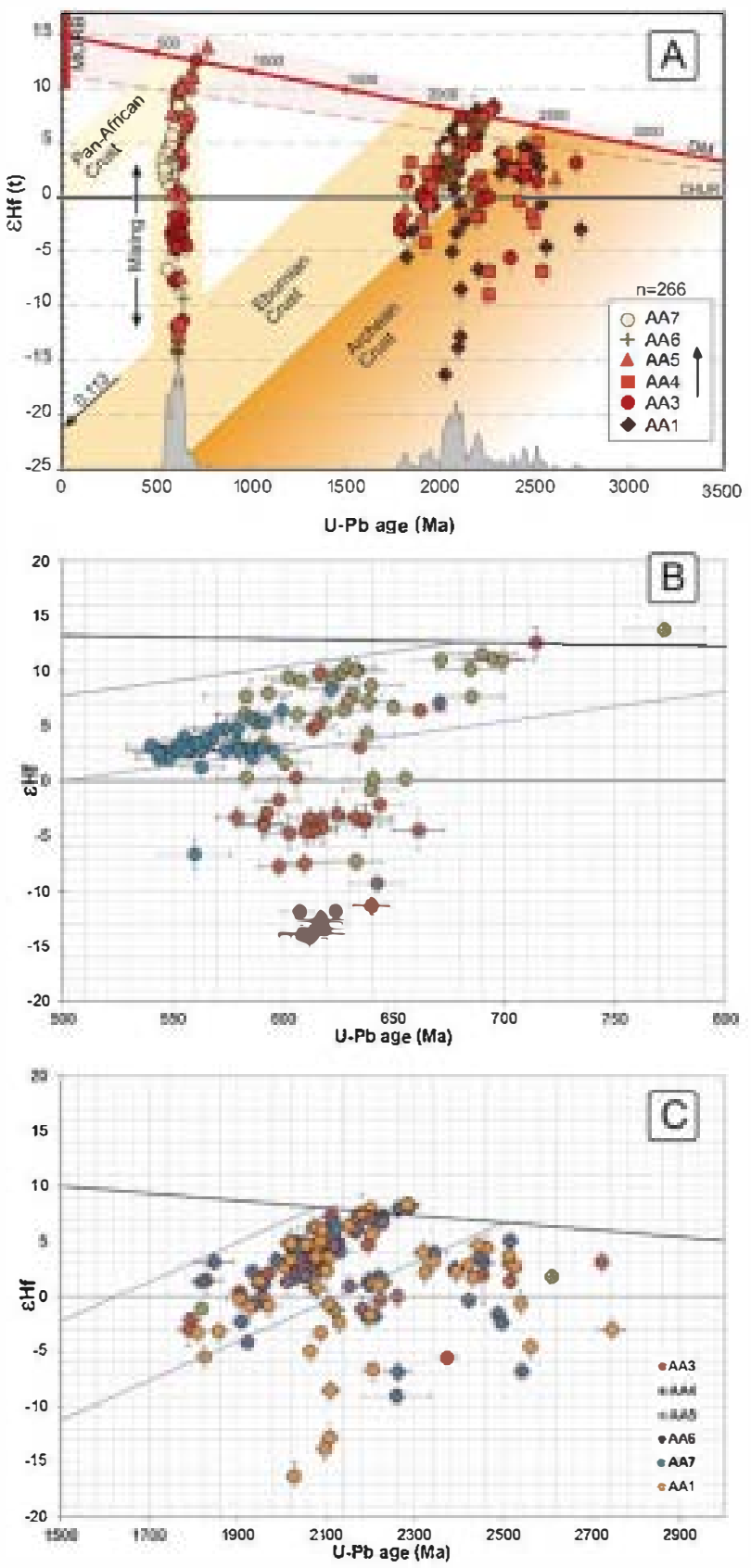

Fig. 3. (a) Hf isotope evolution diagram summarizing the data of zircon from all the rocks studied. The continental crust evolution trends of the main component of the WAC are shown in different colors, and the probability plot of the zircon age populations are represented in grey. See text for discussion. For details and references of depleted mantle evolution see Gerdes and Zeh(2006) and Dhuime et al . (2011). (b) Hf isotope evolution diagram for the "young" (Neoproterozoic zircons). (c) Hf isotope evolution diagram for the "old" zircons. Dat a were calculated using the decay constant of $1.867 \times 10^{-11}$ (Schereret al., 2001), and the CHUR parameters of Bouvier et al. (2008).

group is related with the evolution and dismantling of an ensialic magmatic arc built upon the continental margin of the WAC. The new data presented here strongly support that interpretation. All these features fit well with an origin in a ensialic magmatic arc geodynamic setting, which in turn fits with the general geodynamic scenario of the north Gonwana margin for this age, dominated by a well-documented Neoproterozoic magmatic arc activity( e.g. Nance et al., 2008 and references therein).

\subsection{Juvenile crust formation at ca. $2.3-2.1 \mathrm{Ga}$}

One of the major crustal growth events identified in the WAC is the Eburnian orogeny (e.g. Abouchami et al., 1990). Medium to high grade metamorphic rocks cross-cut by Eburnian (ca. 2.2-2.0 Ma) granitoid batholiths outcrop in several inliers in the Anti-Atlas and form a great part of the Reguibat and Man Shields (Fig. 1a). Geochemical and Nd isotopic studies in the Zenaga Inlier of the Anti-Atlas suggest a juvenile metasedimentary crustal source for some peraluminous granitoids, although the geochemical signature of some parts of the plutons seems strongly modified by a fluid-related reactivation during the Pan-African orogeny (Ennih and Liegeois, 2008). In the studied samples, the zircons of the age interval 2.3-1.79 Ga represent a proportion with respect to the total population between 93\% (sample AA6, sandstone from Azarwas formation) and $3 \%$ in the younger sample (AA7, Ouarzazate group conglomerate). The Hf isotope data of this study confirm the predominantly juvenile origin of the Eburnian rocks, with only minor components of Archean crust involved. About 75\% of the 154 analyzed grains have positive $\varepsilon \mathrm{Hf}_{\mathrm{i}}$ values. Zircons with positive to slightly negative $\varepsilon \mathrm{Hf}_{\mathrm{i}}$ in the age interval $1.79-2.3 \mathrm{Ga}$ arrange in a linear array with a slope of ${ }^{176} \mathrm{Lu} /{ }^{177} \mathrm{Hf}$ of $\sim 0.01$ suggesting a main phase of crustal growth between 2.3 and $2.1 \mathrm{Ga}$ and subsequent recycling of this crust between 2.2 and $1.8 \mathrm{Ga}$ (Fig. 3a and c). This $\varepsilon \mathrm{Hf}_{\mathrm{i}}$-age evolution is consistent with formation of juvenile magmas in an island arc setting, the changeover to a continental arc setting and a subsequent continent-continent collision. While during the first stage only juvenile magmas extracted from a depleted mantle reservoir were formed, the subsequent stages are characterized by increasing amount of reworking of the earlier arc rocks and older crustal components.

Consequently, the evolution of the Eburnian orogeny is apparently dominated by new crustal formation in a magmatic arc environment. The Archean component involved in the Eburnian cycle is revealed by the zircons with negative $\varepsilon \mathrm{Hf}_{\mathrm{i}}$ values, with $\mathrm{T}_{\mathrm{DM}}$ model ages varying between 2.25 and $3.26 \mathrm{Ga}$. The small size of the group of zircons with the lowest $\varepsilon \mathrm{Hf}_{\mathrm{i}}$ suggest that only limited amounts of old Mesoarchean crust (between 3.25 and 3.0) were reworked during the Eburnian cycle, and the zircons with intermediate epsilon values indicate mixing of the Archean precursors with variable amounts of the Eburnian arc rocks.

\subsection{Lower Paleoproterozoic and Neoarchean evolution $(2.3-2.75 \mathrm{Ga})$}

The oldest group of zircons, between 2.3 and $2.75 \mathrm{Ga}$, is form a minor age population represented in all samples except in AA6 and AA7. They are a small proportion in samples AA3 and AA5 ( $1 \%$ and $5 \%$ ), whereas they reach a significant proportion, $17 \%$ and $16 \%$, in samples AA1 and AA4, respectively (Fig. 3a). The meaning of these ages is not clear, because no tectonothermal events in that range of ages have been described in the WAC up to now (except for the older part of the range). The youngest granitoids related with the Neoarchean development of the Reguibat shield are $2726 \pm 7 \mathrm{Ma}$ (Potrel et al., 1998; Key et al., 2008), and the following registered events are the early Eburnian ca. $2.25 \mathrm{Ga}$. A gap in zircon ages between ca. 2.7 and $2.2 \mathrm{Ga}$ is assumed to be characteristic of rocks coming from the WAC (Nance et aI., 2008). Abati et al. (2010) suggested that the presence of this group of zircons in these rocks indicates that there could be a tectonothermal event in the northern part of the WAC with a peak at ca. 2.5-2.4 Ga, which has not been described yet. The Hf model ages of this zircons lie between 2.38 and $3.16 \mathrm{Ga}$, and $75 \%$ of $\varepsilon \mathrm{Hf}_{\mathrm{i}}$ values are positive. Although the values are not so close to the depleted mantle array than those of the previous group, their main isotopic component is also juvenile, mixed with variable proportions of reworked Archean crust. Thus, 
this hypothetic event can be associated with reworking of a juvenile crust with only moderate amounts of Archean crust involved. -ther possibility is that this group of zircons is far-traveled and come from an unknown source, but at this respect only speculations can be made.

\section{Summary and conclusions}

The Hf isotope record of detrital zircons from Neoprotezoroic sequences of the Anti-Atlas gives some clues about the nature of the major geologic events forming the WAC and their crustal evolution. Three major groups of zircon ages point to the existence of three peaks of TTG production. The three groups have predominant juvenile signatures, and their $T_{\mathrm{DM}}$ ages indicate the moment of extraction of crustal domains from the mantle. Other isotopic components suggest variable amounts of reworking of older crust:

1. The Pan-African evolution is dominated by juvenile crust formation, as is suggested by the high proportion of zircons with positive epsilon values (more than 70\%). The juvenile character is linked with the evolution of a magmatic arc developed in the north margin of the WAC, identified with the long-lived Cadomian-Avalonian arc activity of the North Gondwana margin. Reworking of the Eburnian crust is indicated by zircons with low $\varepsilon \mathrm{Hf}_{\mathrm{i}}$ values and $\mathrm{T}_{\mathrm{DM}}$ ages between 1.85 and $2.0 \mathrm{Ga}$ and interaction and mixing between this old crust and the juvenile magmas is indicated by zircons with intermediate epsilon and $T_{\mathrm{DM}}$ ages.

2. The major crustal growth event of 2.3-2.1 Ga typical of the WAC also involves predominance of juvenile crust formation, mixed with variable proportions of reworked Archean crust. The $\varepsilon \mathrm{Hf}_{\mathrm{i}^{-}}$ age evolution is consistent with a first magmatic episode in an island arc setting, the changeover to a continental arc setting and a subsequent continent-continent collision.

3. The Lower Paleoproterozoic and Neoarchean evolution involves a group of detrital zircon ages (described by Abati et al., 2010) that has not been identified in the igneous or metamorphic rocks of the north WAC basement. Their Hf isotope signature points to reworking of juvenile crust mixed with moderate amounts of Archean crust. The significance of these ages is uncertain: they can represent a tectonothermal event not discovered yet in the Reguibat Shield or the zircons can be far traveled from an unknown source.

\section{Aclnowledgements}

Field work was funded by project A-7287-06 from the Agencia Española de Cooperación Internacional (AECI), and analytical work was funded by project CM-UCM-910129 from the Comunidad de Madrid.

\section{Appendix A. Supplementary data}

Supplementary data associated with this article can be found, in the online version, at http:/dx.doi.or/10.1016/ j.precamres.2012.06.005.

\section{References}

Abati, J., Arenas, R., Martínez Catalán, J.R., Díaz García, F., 2003. Anticlockwise P-T path of granulites from the MonteC astelo Gabbro (Ordenes Complex NW Spain). Journal of Petrology 44 (2), 305-327.

Abati, J., Aghzer, A.M., Gerdes, A., Ennih, N., 2010. Detrital zircon ages of Neoproterozoic sequences of the Moroccan Anti-Atlas belt. Precambrian Research 181 (1-4), 115-128

Abouchami, W., Boher, M., Michard, A ., Albarede, F., 1990. A major 2.1 Ga event of mafic magmatism in West Africa: an early stage of crustal accretion. Journal of Geophysical Research 95, 17605-17629.
Aït Malek, H., Gasquet, D ., Bertrand,J.M., Leterrier, J., 1998. Eburnian and Pan african granitoids from the Igherm, Kerdous and Bas-Draa Proterozoic inliers (western Anti-Atlas, Morocco): U-Ph geochronology on zircon. Comptes Rendus de l'Academie des Sciences Series IIA Earth and Planetary Science 327. 819-826.

Amelin, Y., Lee, D.C., Halliday, A.N., 2000. Early-middle archaean crustal evolution deduced from Lu-Hf and U-Pb isotopic studies of single zircon grains. Geochimica et CosmochimicaActa 64 (24), 4205-4225.

Andersen, T., Griffin, W.L., Pearson, N.J., 2002. Crust al evolution in the SW part of the baltic shield: the Hf isotope evidence. Joumal of Petrology 43 (9), 1725-1747.

Auvray, B. Peucat, J-J . Potrel, A Burg. J-P Caruba, C., Dars, R, Lo, K, 1992. Données géochronologiques nouvelles sur l'Archéen de l'Amsaga (Dorsale Réguib at Mauritanie). Comptes Rendus de l'Académie des Sciences 315 (1), 63-70.

Boher, M., Abouchami, W., Michard,A., Albarede, F.,Arndt, N.T., 1992. Crustal growth in West Africa at 2.1 Ga. Journal of Geophysical Research 97, 345-369.

Bouvier,A., Vervoort,J.D., Patchett,P.J., 2008. The Lu-Hf and Sm-Nd isotopic composition of CHUR: constr aints from unequilibrated chondrites and implications for the bulk composition of terrestrial planets. Earth and Planetary Science Letters $273(1-2), 48-57$

De Wall, H., Kober, B., Greiling, R.O., Errami, E., Ennih, N., 2001. Age and structural setting of the grantoid emplacement in the area of Imiter (Eastern Saghro). In: Abstrac, $2^{\text {nd }}$ Coll. 3M, Marrakech, p. 19.

Dhuime, B., Hawkesworth, C., Cawood, P., 2011. When continents formed. Science 331 (6014), 154-155.

D'Lemos, R.S., Inglis, J.D., Samson, S.D ., 2006. A newly discovered orogenic event in Morocco: Neoproterozic ages for supposed Eburneanbasement of the Bou Azzer inlier* Anti-Atl as Mountains. Precambrian Research 147 (1-2), 65-78.

El Hadi, H., Simancas, J.F., Marúnez-Poyatos, D., Azor, A., Tahiri, A., Montero, P., Fanning, C.M., Bea, F., González-Lodeiro, F., 2010. Structural and geochronological constraints on the evolution of the Bou Azzer Neoproterozoic ophiolite (Anti-Atlas, Morocco). Precambrian Research 182 (1-2), 1-14

Ennih, N., Liegeois,J.-P., 2008. The boundaries of theWest African craton, with special reference to the basement of the Moroccan metacratonic Anti-Atlas belt. Geological Society, London, Special Publications 297 (1), 1- 17

Frei, D., Gerdes,A ., 2009. Precise and accurate in situ U-Pb dating of zircon with high sample throughput by automated IA-SF-ICP-MS. Chemical Geology 261 (3-4), $261-270$.

Gasquet, D., Levresse, G., Cheilletz, A., Azizi-Samir, M.R., Mouttaqi, A., 2005. Contribution to a geodynamic reconstruction of the Anti-Atlas (Morocco) during Pan-African times with the emphasis on inversion tectonics and metallogenic activity at the Precambrian-Cambrian transition. Precambrian Research 140 (3-4), 157-182.

Gasquet, D., Ennih, N., Liégeois,J.P.,Soulaimani,A., Michard,A., 2008. The Pan-African Belt. Continental Evolution: The Geology of Morocco, pp. 33-64.

Gerdes, A.,Zeh, A., 2006. Combined U-Ph and Hf isotope l A-(MC-)ICP-MS analyses of detrital zircons: comparison with SHRIMP and new constraints for the provenance and age of an Armorican metasediment in Central Germany. Earth and Planetary Science Letters 249 (1-2), 47-61.

Gerdes, A., Zeh, A., 2009. Zircon formation versus zircon alter ation-new insights from combined U-Ph and Lu-Hf in situ lA-ICP-MS analyses, and consequences for the interpretation of Archean zircon from the Central Zone of the Limpopo Belt. Chemical Geology 261 (3-4), 230-243.

Griffin, W.L., Belousova, E.A., Shee, S.R., Pearson, N.J., O'Reilly, S.Y., 2004. Archean crustal evolution in the northern Yilgarn Craton: U-Ph and Hf-isotope evidence from detrital zircons. Precambrian Research 131 (3-4),231-282.

Hefferan, K.P., Admou, H., Karson, J.A., Saquaque,A., 2000. Anti-Atlas (Morocco) role in Neoproterozoic Western Gondwana reconstruction. Precambrian Research $103,89-96$

Hirdes, W., Davis, D.W.,Eisenlohr, B.N., 1992. Reassessment of Proterozoicgranitoid ages in Ghana on the basis of U/Ph zircon and monaite dating. Precambrian Research 56 (1-2), 89-96.

Hurley, P.M., Leo, G.W., White, R.W., Fairbaim, H.W., 1971. Liberian age province (about 2700 m.y.) and ad jacent provinces in Liberia and Sierra Leone. Geological Society of America Bulletin 82 (12), 3483-3490.

Inglis, J.D., MacLe an, J.S., Samson, S.D., D’Lemos, R.S., Admou, H., Hefferan, K., 2004 A precise U-Pb zircon age for the Bleïda granodiorite, Anti-Atlas, Morocco: implications for the timing of deformation and terrane assembly in the eastern Anti-Atlas. Journal of African Earth Sciences 39 (3-5), 277-283.

Janousek, V., Gerdes, A., Vrana, S., Finger, F., Erban, V., Friedl, G., Br aithwaite, C.J.R. 2006. Low-pressure Granulites of the Lisov Massif, Southern Bohemia: Visean Metamorphism of Late Devonian PlutonicArcRocks.J. Petrology 47 (4), 705-744.

Key, R.M., Loughlin, S.C., Gillespie, M., Del Rio, M., Horstwood, M.S.A., Crowley, Q.G. Darbyshire, D.P.F., Pitfield, P.E.J., Henney, P.J., 2008. Two mesoarchae an terranes in the Reguibat shield of NW Mauritania. Geological Society, London, Special Publications 297 (1), 33-52.

Kröner, A., Ekwueme, B.N., Pidgeon, R.T., 2001. The oldest rocks in West Africa: SHRIMP zircon age for early archean migmatitic orthogneiss at Kaduna, Northern Nigeria. The Journal of Geology 109 (3), 399-406.

Leblanc, M., Lancelot, J.R., 1980. Interprétation géodynamique du domaine panafricain (Précambrien terminal) de l'Anti-Atlas (Maroc) à partir de données géologiques et géochronologiques. Canadian Journal of Earth Sciences 17 (1), $142-155$

Levresse, G., Azizi, M.S., Cheilletz, A., Gasquet, D., Zyadi, R., Ennaciri, D., Archibald, D., Ouguir, H., 2001. Le gisement Ag-Hg d'lmiter(Anti-Atlas, Maroc): Nouvelles donneés U-Ph, ${ }^{40} \mathrm{Ar}{ }^{39} \mathrm{Ar}{ }^{*}{ }^{34}$ Set microstructurales appuy ant le modéle génétique hydrothermal-épithermal. ln: Abstract 2nd Coll. 3M, Marrakech, p. 97. 
Liégeois, J.P., Claessens, W., Camara, D., Klerkx,J., 1991. Short-lived Eburnian orogeny in southern Mali. Geology, tectonics, U-Pb and Rb-Sr geochronology. Precambrian Research 50 (1-2), 111- 136.

Morag, N.,Avigad, D., Gerdes, A., Belousova, E., Harlavan, Y., 2011. Crustal evolution and recycling in the northern Arabian-Nubian shield: new perspectives from zircon Lu-Hf and U-Pb systematics. Precambrian Research 186 (1-4), 101-116.

Nance, R.D., Murphy, J.B., Strachan, R.A., Keppie, J.D., Gutierrez-Alonso, G., Fernandez-Suarez, J., Quesada, C., Linnemann, U., D’lemos, R., Pisarevsky, S.A., 2008. Neoproterozoic-early Palaeozoic tectonostratigraphy and palaeogeography of the peri-Gondwanan terranes: Amazonian v. West African connections. Geological Society, London, Special Publications 297 (1), 345-383.

Nebel-Jacobsen, Y., Müinker, C., Nebel, O., Gerdes, A., Mezger, K., Nelson, D.R., 2010. Reworking of Earth's first crust: constraints from Hf isotopes in Archean zircons from Mt. Narryer, Australia. Precambrian Research 182 (3), 175- 186.

Patchett, P.J., Kouvo, O., Hedge, C.E., Tatumoto, M., 1982. Evolution of continental crust and mantle heterogeneity: evidence from Hf isotopes. Contributions to Mineralogy and Petrology 78 (3), 279-297.

Potrel, A., Peucat, J.J., Fanning, C.M., Auvray, B., Burg, J.P., Caruba, C., 1996. 3.5 Ga old terranes in the West African Craton, Mauritania. Journal of the Geological Society 153 (4), 507-510.

Potrel, A., Peucat, J.J., Fanning, C.M., 1998. Archean crustal evolution of the West African Craton: example of the Amsaga Area (Reguibat Rise) U-Pb and Sm-Nd evidence for crustal growth and recycling. Precambrian Research 90, 107-117.

Rocci, G., Bronner, G., Deschamps, M. (Eds.), 1991. Ciystalline Basement of the West African Craton. The West African Orogens and Circum-Atlantic Correlatives. Springer-Verlag, Berlin, pp. 31-61.

Samson, S.D., Inglis, J.D., D’Lemos, R.S., Admou, H., Blichert-Toft, J., Hefferan, K., 2004. Geochronological, geochemical, and Nd-Hf isotopic constraints on the origin of
Neoproterozoic plagiogranites in the Tasriwine ophiolite, Anti-Atlas orogeny, Morocco. Precambrian Research 135 (1-2), 133- 147.

Saquaque,A., Admou, H., Karson, J., Hefferan, K., Reuber, I., 1989. Precambrian accretionary tectonics in the BouAzzer-El Graara region, Anti-Atlas, Morocco. Geology 17 (12), 1107-1110.

Scherer, E., Mïnker, C., Mezger, K., 2001. Calibration of the Lutetium-Haf nium clock. Science 293 (5530), 683-687.

Schofield, D.I., Horstwood, M.S.A., Pitfield, P.E.J., Crowley, Q.G., Wilkinson, A.F., Sidaty, H.C.O., 2006. Timing and kinematics of Eburnean tectonics in the central Reguibat shield, Mauritania. Journal of the Geological Society 163 (3),549-560.

Stevenson, R.K., Patchett, P.J., 1990. Implications for the evolution of continental crust from Hf isotope systematics of Archean detrital zircons. Geochimica et Cosmochimica Acta 54 (6), 1683-1697.

Thiéblemont, D., Goujou, J.C., Egal, E., Cocherie,A., Delor, C., Lafon, J.M., Fanning, C.M. 2004. Archean evolution of the Leo Rise and its Eburnean reworking. Journal of African Earth Sciences 39 (3-5), 97-104.

Thomas, R.J., Chevallier, L.P., Gresse, P.G., Harmer, R.E., Eglington, B.M., Armstrong, R.A., de Beer, C.H., Martini, J.E.J., de Kock, G.S., Macey, P.H., Ingram, B.A., 2002 Precambrian evolution of the Sirwa Window, Anti-Atlas Orogen, Morocco. Precambrian Research 118 (1-2), 1-57.

Walsh, G.J., Aleinikoff, J.N., Benziane, F., Yazidi, A., Armstrong, T.R., 2002. U-Pb zircon geochronology of the Paleoproterozoic Tagragra de Tata inlier and its Neoproterozoic cover, western Anti-Atlas, Morocco. Precambrian Research 117. $1-20$.

Zhang, S.-B., Zheng, Y.-F., Wu, Y.-B., Zhao, Z.-F., Gao, S., Wu,F.-Y., 2006. Zircon U-Pb age and $\mathrm{Hf}$ isotope evidence for $3.8 \mathrm{Ga}$ crustal remnant and episodic reworking of Archean crust in South China. Earth and Planetary Science Letters 252 (1-2), 56-71. 